\title{
Microscopic Structural Features of Water in Aqueous-Reline Mixture of Varying Composition
}

\author{
Soham Sarkar, Atanu Maity, and Rajarshi Chakrabarti* \\ Department of Chemistry, Indian Institute of Technology Bombay, Powai, Mumbai-400076 \\ E-mail: rajarshi@chem.iitb.ac.in
}

\begin{abstract}
Reline, a mixture of urea and choline chloride in 2:1 molar ratio, is one of the most frequently used deep eutectic solvents. Pure reline and its aqueous solution have large scale industrial use. Owing to the presence of active hydrogen bond formation sites, urea and choline cation can disrupt the hydrogen-bonded network in water. However, a quantitative understanding of the microscopic structural features of water in the presence of reline is still lacking. We use extensive all-atom molecular dynamics simulations to elucidate the effect of the gradual addition of co-solvents on microscopic arrangements of water molecules. We consider four aqueous solutions of reline, between the wt\% 26.3 to 91.4. A disruption of the local hydrogenbonded water structure is observed on inclusion of urea and choline chloride. The extent of deviation of water structure from tetrahedrality is quantified using the orientational order parameter $\left(q_{t e t}\right)$. Our analyses show a monotonic increase in structural disorder as the co-solvents are added. Increment in the $q_{\text {tet }}$ values are observed when highly electro-negative hetero-atoms like Nitrogen, Oxygen of urea and choline cations are counted as the partners of the central water molecules. Further insights are drawn from the characterization of the hydrogen-bonded network of the water and we observe gradual rupturing of waterwater hydrogen bonds and its subsequent replacement by the water-urea hydrogen bonds. A negligible contribution from the hydrogen bonds between water and bulky choline cation has also been found. Considering all the constituents as the hydrogen bond partner we calculate the possibility of successful hydrogen bond formation with a central water molecule. This gives a clear picture of the underlying mechanism of water replacement by urea.
\end{abstract}




\section{Introduction}

Choline chloride $(\mathrm{ChCl})$ based deep eutectic solvents (DES) have become a designer solvent owing to their appealing properties and benign nature. ${ }^{1-10}$ The microscopic arrangement and properties of DES can be tailored by changing the molecular ratio of the constituent molecules. Low cost, easy handling, and low toxicity have enabled DES to emerge as a promising solvent media in the field of separation, synthesis, catalysis, and electrochemical process. ${ }^{11-14}$ Its remarkable physical properties and biocompatibility have caught the attention of various research groups leading to its wide-spread applications in diverse research fields, including the preservation of enzyme/protein activity. ${ }^{15,16}$ Most commonly used hydrogen bond donors like urea, ethylene glycol, malonic acid, etc. are taken in a specific ratio with $\mathrm{ChCl}$ to obtain these lower melting point mixtures. ${ }^{17-20}$ Several studies related to the morphological properties of ionic liquids (IL) guide the community regarding the wise use of the solvent, which is largely lacking in the case of DES. ${ }^{21-27}$

Reline is one of the most celebrated and highly used DESs, where urea $\left(\mathrm{H}_{2} \mathrm{~N}-\mathrm{CO}-\mathrm{NH}_{2}\right)$ is used as the hydrogen bond donor with choline chloride $\left(\left[\left(\mathrm{CH}_{3}\right)_{3} \mathrm{~N}-\left(\mathrm{CH}_{2}\right)_{2}-\mathrm{OH}\right]^{+} \mathrm{Cl}^{-}\right)(\mathrm{ChCl})$ in 2:1 molar ratio. ${ }^{28,29}$ Apart from its biodegradability and economic viability, ${ }^{17,28}$ reline also has ample industrial applications. ${ }^{30-34}$ In particular reline has profound use in surface coating, biodiesel synthesis, and enzymatic reaction. ${ }^{35-37}$ Owing to its non-reactivity towards water, and acceptable toxicity limit, reline is used as a solvent for poorly water-soluble drugs. ${ }^{38}$ Recently, Monhemi and co-workers ${ }^{39}$ pointed out lower diffusivity of highly hydrogen-bonded urea in the proximity of Candida Antarctica lipase B that causes structural protection of the enzyme in the presence of $\mathrm{ChCl}$ forming a deep eutectic solvent. A similar kind of ultraslow solvent/cosolvent dynamics in the presence of reline is also established in our recent work, that effectively hinders the protein to deviate much from its equilibrium position. ${ }^{40}$ In this earlier work, we thoroughly analyzed the reline mediated structural protection of model protein HP-36. ${ }^{41}$ FTIR $^{42}$, time-resolved fluorescence spectroscopy ${ }^{6}$ have also been used to explore the stabilizing mechanism of reline. Choline cation has been combined with negatively charged amino acids to form a new class of IL, which has no environmental effects. $^{43,44}$ Sahoo et al. in an experimental and computational approach, showed an enhanced stability of Cytochrome-c (Cyt-c) in the presence of amino acid-based biodegradable choline IL. ${ }^{45}$ Reline is also found to protect C-kit G-Quadruplex DNA from severely high temperature. ${ }^{46}$ Hydrogen bond mediated electrostatic interaction plays a pivotal role in stabilizing reline microstructure. ESPR, Neutron scattering, and molecular dynamics studies establish the position of the chloride ion at the closer proximity of urea and hydroxyl group of choline cation. ${ }^{19,47,48}$ The common outcome of all these studies is the hydrogen bond formation between the chloride ion and the urea molecule, which in turn accommodate the urea within the hydrogen bond network. Posada et al. confirmed an inhomogeneity in the structure of reline, despite the 
presence of the hydrophilic choline chloride and urea molecules. ${ }^{49}$ However, only few experimental ${ }^{49-52}$ and simulation studies ${ }^{53}$ demonstrated the role of the addition of water on the hydrogen-bonded network of reline. It has been shown that the viscosity, density, and refractive index of reline-water mixture are heavily disturbed $^{50}$ In a recent work, Kashyap and group ${ }^{54}$ have reported a gradual modification of hydrogen bonds between the constituents of reline and water and found a drastic structural change of reline above the addition of $40 \mathrm{wt} \%$ of water, which is in agreement with the neutron scattering study by Edler and coworkers. ${ }^{52}$ In another work, Kashyap and group have incorporated molecular dynamics simulation and reported the strengthening of the hydrogen bond between ethylene glycol and its subsequent nanosegregation on the addition of water and pointed out a competition between choline cation and ethylene glycol in making a hydrogen bond with the anion. ${ }^{55}$ Similar kind of competitive hydrogen bond formation in case of reline is also reported from the first principle molecular dynamics simulation by Siepmann and co-workers..$^{53}$

The formation of three-dimensional hydrogen bonds predominantly makes the water structure tetrahedral. ${ }^{56}$ Conversely, the addition of osmolytes into water has an immense effect on the microscopic structure of water, for example: in a recent work, Smiatek and group proposed the strengthening of the water network in the presence of urea and TMAO. ${ }^{57}$ Besides that TMAO alone could also impact water network. ${ }^{58-63}$ Molecular dynamics simulations have shown that water-TMAO interaction is stronger than that of watertetramethylurea. ${ }^{64}$ This is in line with an earlier study by Paul et al. ${ }^{65}$ Despite having a similar structural framework, tert-butyl alcohol and TMAO show a drastic difference in their behaviors on the water structure. ${ }^{26}$ The presence of multiple potential hydrogen bonding sites is responsible for the interference of urea into the tetrahedral structure of water. ${ }^{58,60,66,67}$ In their investigation, Choudhury and group probed a range of aqueous solutions of urea from $\sim 1 \mathrm{M}$ to $\sim 9 \mathrm{M}$ and verified that even at very high concentration urea does not have its deleterious effect on the tetrahedrality of water. ${ }^{68}$ The same group in another work correlates the structural order and hydrogen bond network of water with varying temperature. ${ }^{56}$ This kind of behavior of urea in water is also reported by Ojha et al. in their recent work ${ }^{69}$, though it is not in agreement with the Raman band analysis experiment by Walrafen et al., ${ }^{70}$ which predicts urea to be a structure breaker. Adding to the case of sustaining tetrahedrality of water around a biomolecule, computational investigations by Bagchi and group have confirmed enhanced structural ordering of water molecules at the minor groove of DNA. ${ }^{71}$ In another study, the extent and mechanism of water structure perturbation in the presence of Glycerol and DMSO is analyzed thoroughly at the interface and the bulk of a nonpolar solute. ${ }^{72}$ Nayar $e t$ $a l$. investigated the degree of disorder of water around a small peptide for different established water models. ${ }^{73}$ Later, Dubey et al. correlate the variation of temperature and contribution of jump-diffusion coefficient with that of the average tetrahedrality of water, which shows a decreasing average tetrahedral 
ordering with increasing temperature, while the contribution of jump-diffusion coefficient increases linearly with that of the average tetrahedral parameter. ${ }^{74}$

The effect of urea on the water structure is arguable and how a bulky molecule like choline affect the structure of water is still largely unknown. In this investigation, by considering a range of aqueous ternary solutions with urea and choline chloride in 2:1 molar ratio, we have explored the role of the individual constituents in making/breaking the tetrahedral framework of water. The extent of contributions of the hetero atoms, capable of forming the hydrogen bond with the central water oxygen, has also been analyzed. The manuscript is arranged as follows: in section 2 we provide the modeling of the system and simulation details, in section 3 we discuss the distribution of solvent and co-solvents. Section 4 comprises of the quantification of the tetrahedrality of water structure and hydrogen bond structure analyses, followed by the concluding remarks in section 5 .

\section{Methods}

\subsection{Modeling}

We construct a cubic simulation box of edge length $\sim 3.6 \mathrm{~nm}$. Subsequently, the simulation box is filled with the solvent and co-solvents accordingly. We have generated five different systems for this investigation, by varying the concentration of co-solvents urea and choline chloride along with the solvent water. These systems are, W: bulk water (represented in orange), W-UC (2:1): aqueous solution of 2M Urea and 1M choline chloride (represented in black), W-UC (4:2): aqueous solution of 4M Urea and $2 \mathrm{M}$ choline chloride (represented in red), W-UC (6:3): aqueous solution of 6M Urea and 3M choline chloride (represented in blue), W-UC (8:4): aqueous solution of $8 \mathrm{M}$ Urea and $4 \mathrm{M}$ choline chloride (represented in green). The aforesaid color codes for these different systems are maintained throughout the manuscript. Detailed information for each system is provided in the supplementary information (Table S1). 


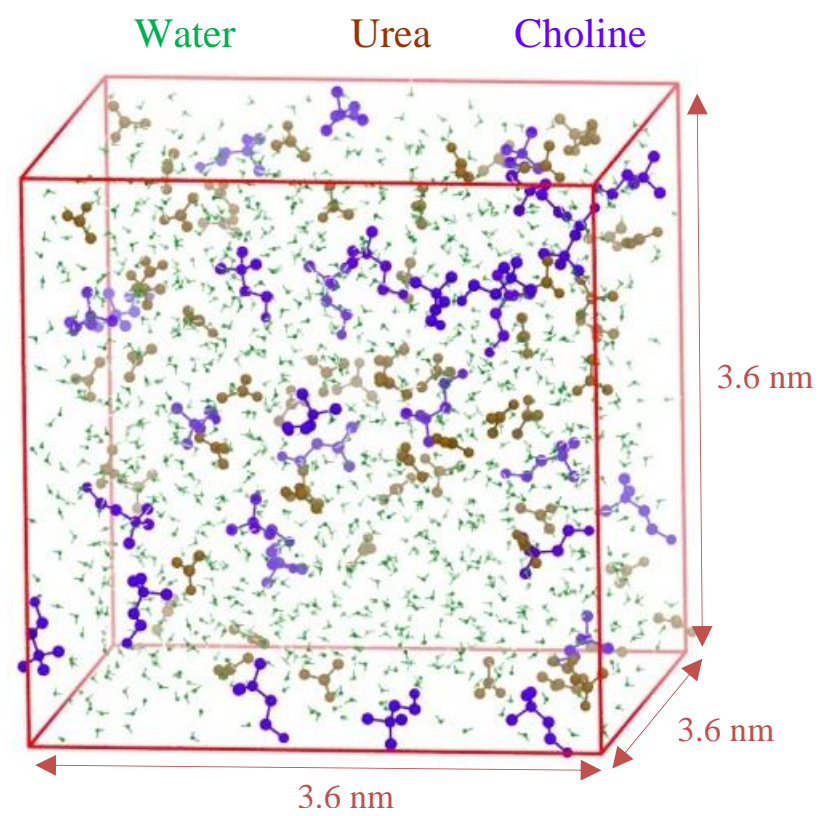

Fig 1: Representative snapshot of the simulation box filled with Water (green), Urea (dark yellow), and Choline (violet). All constituents are represented in ball-stick. For the sake of visual clarity hydrogen atoms and chloride ions are not shown.

\subsection{Simulation Details}

We have performed all-atom molecular dynamics simulations using GROMACS package. ${ }^{75}$ OPLS-AA ${ }^{76}$ force field is used to collect the topological parameters for the ammonium-based protein secondary structure stabilizer choline chloride and the protein denaturant urea, which is consistent with our recent works. ${ }^{40,41,77}$ OPLS-AA performs quite well to reproduce experimentally verified physical properties. For example, in a very recent study, Doherty and Acevedo could reproduce physical properties like density, viscosity, surface tension and heat capacity of $\mathrm{ChCl}$ based DES that included urea too, for a wide range of temperature using the OPLS force field. ${ }^{78}$ Again in a recent work, Zhu et al. also showed that OPLS-AA force field could effectively reproduce the osmotic coefficient of sixteen drug-like small molecules. ${ }^{79}$ The force field parameters of the small molecules used in this investigation are represented elsewhere. ${ }^{41}$ To suffice the purpose of force field validation of the small molecules used here, we have computed the bulk densities of the investigated solutions (Table S2 and Fig S1 in Supplementary Information) and compare it with the experimentally verified one. ${ }^{80} \mathrm{We}$ have found a good agreement between the experimental and simulated bulk density. SPC/E water model is used for our study ${ }^{81}$ A 5000-steps energy minimization is performed for each system using the steepest descent method ${ }^{82}$ to remove the initial steric clashes. Subsequently, a 500 ps equilibration under NVT ensemble is performed using the V-rescale thermostat ${ }^{83}$ to equilibrate each system at a temperature of $310 \mathrm{~K}$ and to avoid the possibility of void formation in the simulation box. Further, we ensure equilibration at isothermal-isobaric (NPT) ensemble by attaining a steady pressure of $\sim 1$ atm using the Parrinello-Rahman barostat ${ }^{84}$, considering a pressure relaxation time of $1 \mathrm{ps}$. We found that this period of equilibration is sufficient for the convergence of thermodynamic parameters like pressure, 
temperature. Short-range Lennard-Jones interactions are accounted for using the minimum image convention. ${ }^{85}$ To estimate the non-bonding interactions including electrostatic as well as van der Waals interactions, a spherical cut-off distance of $1 \mathrm{~nm}$ is taken into account. In all three directions, periodic boundary conditions have been used to remove the edge effects. SHAKE algorithm is incorporated to constrain the bonds involving the hydrogen atom of the water molecules. ${ }^{86}$ Long-range electrostatic interactions are calculated using the particle mesh Ewald (PME) method. ${ }^{87}$ The production run time considered for all ternary mixture systems is $100 \mathrm{~ns}$ (for bulk water it is 50ns) with a time step of $2 \mathrm{fs}$ and the trajectory is saved at every $2 \mathrm{ps}$ for analysis purpose. To extract a wide range of microscopic structural properties of the solvent, in-built modules of GROMACS 5.0.5 and some in-house scripts are used. For visualization purpose, we have used VMD 1.9.3. ${ }^{88}$

\section{Distribution of solvent and co-solvents around Water}

\subsection{Radial Distribution Function}

Radial distribution function (rdf) $(\mathrm{g}(\mathrm{r}))$ provides insights on the average picture of the distribution of the constituents in the systems considered. This is calculated with the in-built module of GROMACS. In this investigation, we are mainly interested in structural aspects of water in the aqueous solution of reline, e.g. its self-distribution and also the distribution of urea and bulky choline molecules around the water-oxygen atoms $(\mathrm{Ow}) . \mathrm{g}(\mathrm{r})$ of water-oxygen $(\mathrm{Ow})$, urea and, choline around water-oxygen $(\mathrm{Ow})$ is represented in Fig 2. We have calculated the $\mathrm{g}(\mathrm{r})$ from the last $10 \mathrm{~ns}$ of the total trajectory. 

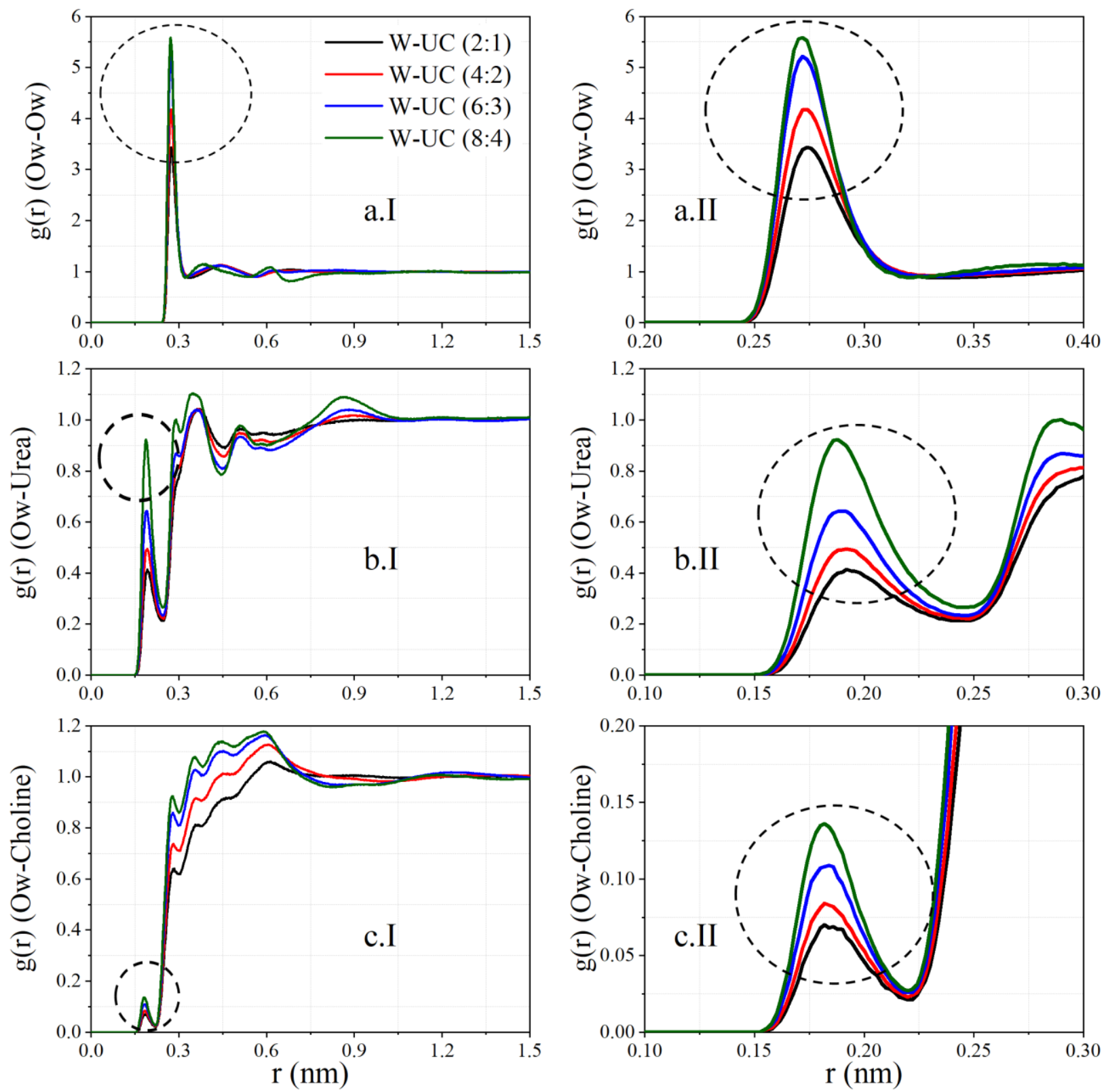

Fig 2: Radial distribution functions $(\mathrm{g}(\mathrm{r})$ ) of a) water-oxygen $(\mathrm{Ow}), \mathrm{b})$ urea, and c) choline around water-oxygen (Ow) for different systems under study. The relevant parts of the aforesaid respective plots are magnified and shown in the same row.

A careful examination of Fig 2 shows the existence of a sharp and single peak $\sim 0.275 \mathrm{~nm}$ (represented in Fig 2.a.I and magnified in Fig 2.a.II) which is present for all systems for the distribution of Ow around Ow. The position of this particular peak is also verified earlier by other groups. ${ }^{89,90}$ While increasing the wt $\%$ of reline in the system (W-UC (2:1) to W-UC (8:4)), water-oxygen atoms (Ow) approach each other as evident from Fig 2.a.II and the g(r) value increases to almost double from W-UC (2:1) to W-UC (8:4). This could be attributed to the crowding induced by reline on the water molecules. Similarly, an increase in the amount of urea (Fig 2.b.I) and bulky choline molecules (Fig 2.c.I) increases their amount around the Ow atoms. A 
closer observation of the $\mathrm{g}(\mathrm{r})$ plots reveals both urea (Fig 2.b.II) and choline (Fig 2.c.II) to have a peak around $0.2 \mathrm{~nm}$, which precedes the Ow-Ow peak. This observation is in agreement with the work by Banerjee et $a l .{ }^{91}$, where the authors show that owing to hydrogen bond formation between water-oxygen and the hydrogen bond donor, there is a peak around $0.2 \mathrm{~nm}$. Here too, we have considered the center of mass (COM) rdf for urea and choline around Ow, where both molecules have potential hydrogen bond donor sites and $\mathrm{Ow}$ is an acceptor. For a deeper analysis of the distribution, we have plotted the $\mathrm{g}(\mathrm{r})$ of Ow/Urea/Choline around Ow for each system individually (Fig S2 in Supplementary Information) where peak intensity and peak positions of the constituents for a particular system are clearly visible.

Further insights come from the quantification of the positional occupancy by the aforesaid components around Ow. We have calculated the relative number fraction $\left(\Phi_{i}(r)\right)$ of Ow/Urea/Choline around Ow to account for how the environment around Ow is being occupied by these constituents as we did in our earlier work. ${ }^{40} \Phi_{i}(r)$ is formulated as-

$$
\Phi_{i}(r)=\frac{N_{i}(r) / \sum_{i} N_{i}(r)}{N_{i}\left(r_{\text {Bulk }}\right) / \sum_{i} N_{i}\left(r_{\text {Bulk }}\right)}
$$

Here, $N_{i}(r)$ signifies the coordination number of Ow/Urea/Choline around Ow and $N_{i}\left(r_{B u l k}\right)$ is designated as the coordination number of Ow/Urea/Choline around Ow at the bulk and $r_{B u l k} \gg 3 \AA ̊$. The subscript $i$ denotes the different components considered in the calculation, here Ow, urea, and choline. 

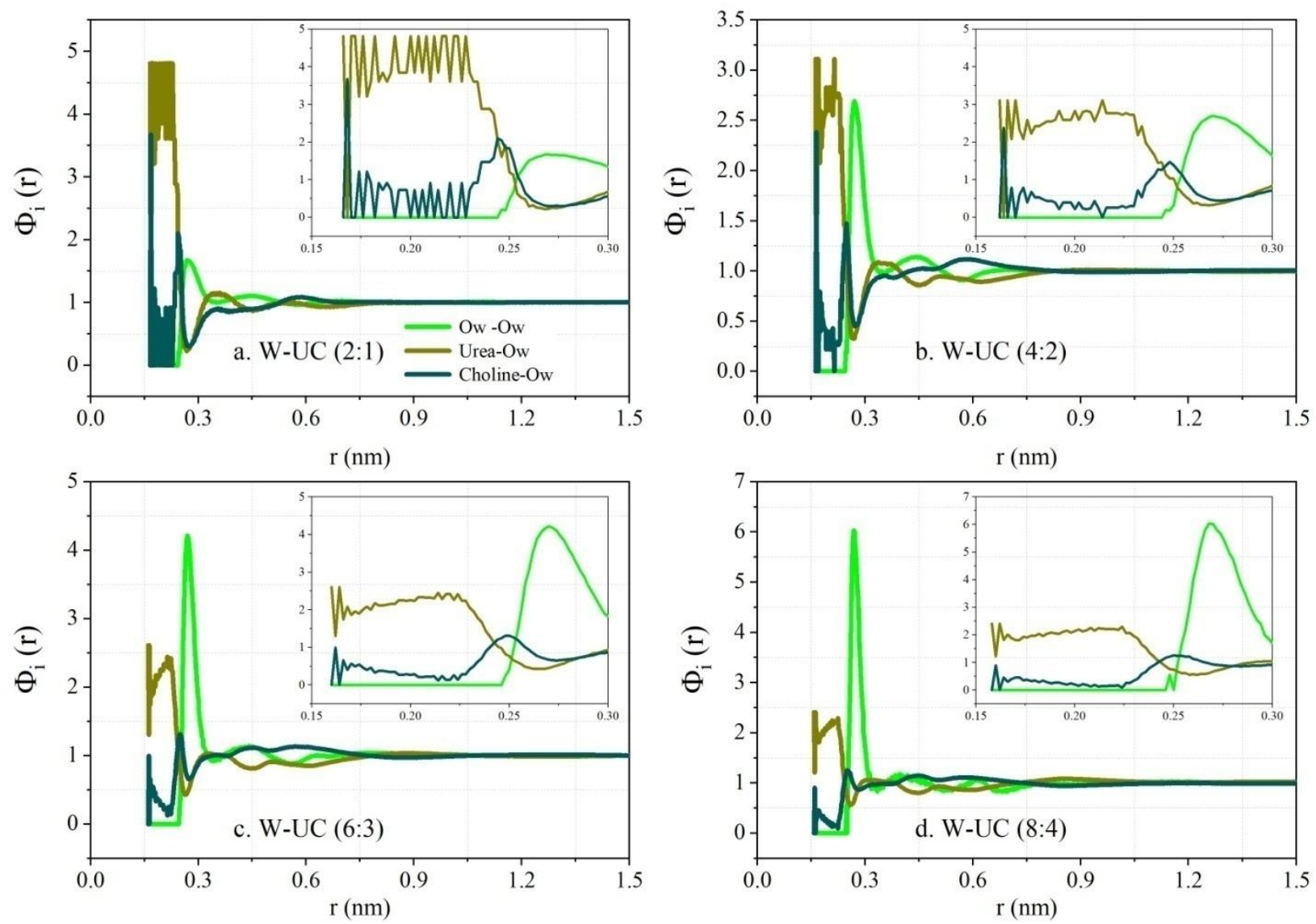

Fig 3: Relative number fraction $\Phi_{i}(r)$ of Ow/urea/choline in (a) W-UC (2:1), (b) W-UC (4:2), (c)W-UC (6:3), (d) W-UC (8:4) as a function of distance (r) from Ow, respectively. In the inset, respective plots are magnified (up to $\mathrm{r}=0.3 \mathrm{~nm}$ ) for better visualization.

$\Phi_{i}(r)>1$ signifies accumulation and $\Phi_{i}(r)<1$ signifies exclusion of a particular constituent. As can be seen from Fig 3, for each system, $\Phi_{i}(r)$ derived from Urea-Ow distribution and Choline-Ow distribution is compensating each other up to $0.25 \mathrm{~nm}$ and after that, there is a decrease of these two $\Phi_{i}(r)$ values and rise of the $\Phi_{i}(r)$, derived from Ow-Ow radial distribution which is also reflected in the g(r) plots (Fig S2 in Supplementary Information). The $\Phi_{i}(r)$ corresponding to Ow-Ow distribution is anticorrelated to $\Phi_{i}(r)$ of Ow-Urea and Ow-choline. This is particularly visible around the maximum of the Ow-Ow distribution, where Ow-Urea and Ow-choline have sharp minima. For each pair, as bulk is approached, preferential accumulation/exclusion is diminished and $\Phi_{i}(r)$ converges to 1 .

\section{Quantification of structural changes of water}

\subsection{Tetrahedral order parameter (TOP)}

The most widely used order parameter to elucidate the microscopic tetrahedral structure of water is the orientational tetrahedral order parameter $\left(q_{\text {tet }}\right)^{92-94}$ (TOP), where $q_{\text {tet }}$ is formulated as- 


$$
q_{t e t}=1-\frac{3}{8} \sum_{j=1}^{3} \sum_{k=j+1}^{4}\left(\cos \theta_{j k}+\frac{1}{3}\right)^{2}
$$

As tetrahedrality is concerned, for neat liquid water, four immediate water-oxygen $(\mathrm{Ow})$ is considered around a central water-oxygen $(\mathrm{Ow})$ and the angle subtended by two partners $(j, k)$ with the central $\mathrm{Ow}$ is designated as $\theta_{j k}$ here. $q_{t e t}=1$ signifies a perfectly tetrahedral ice-like structure, and $q_{t e t}=0$ signifies a completely random orientation like an ideal gas. A detailed explanation of this formula can be found elsewhere ${ }^{95,96}$ For calculation purposes we have considered the last $10 \mathrm{~ns}$ of the trajectory.

\subsubsection{Probability distribution of TOP}

Following the trend found in some recent works, ${ }^{68,72,97}$ we have calculated $\boldsymbol{q}_{\text {tet }}$ using two protocols. In Protocol-1, while calculating $\boldsymbol{q}_{\boldsymbol{t e t}}$, only four immediate Ow atoms are chosen around a central Ow as the partners, and in Protocol-2 other electronegative heavy atoms (Nitrogen and Oxygen from Urea and Choline) along with Ow are chosen as the partners.

As can be seen from Fig 4, the probability distribution of TOP $\left(P\left(q_{t e t}\right)\right)$ of bulk water is represented in orange for comparison purpose (with Protocol-1 and Protocol-2), which has its peak value at $q_{t e t} \sim 0.75$. The first row of Fig 4 is dedicated to Protocol-1 (solid lines) and the second row is dedicated to Protocol-2 (dashed). It is evident from Fig 4 that for both Protocol-1 and 2 a shift of peak towards lower $q_{\text {tet }}$ is observed as wt $\%$ of reline is increased, which indicates deviations from tetrahedrality and existence of structural disorderness. The peaks for the W-UC (2:1), W-UC (4:2), and W-UC (6:3) resides between $q_{\text {tet }}$ value of 0.25 to 0.5 . This is to be noted that in the case of W-UC (8:4) (for Protocol-1 and Protocol-2) the distribution is broad without any distinct peaks indicating the absence of any preferred structure. At this point, the increasing deviations of water molecules from tetrahedrality with increased reline wt $\%$ may seem contradictory with the increasing peak height in Ow-Ow rdf (Fig 2a). This apparent contradiction arises because, at higher concentrations, cosolvent molecules impose closer packing to the water molecules but the proper orientation to attain tetrahedral geometry is hindered in the crowded environment. Similar behavior was observed by Elola, M. D. et al. in aqueous solutions of formamide of different concentrations. ${ }^{98}$ 

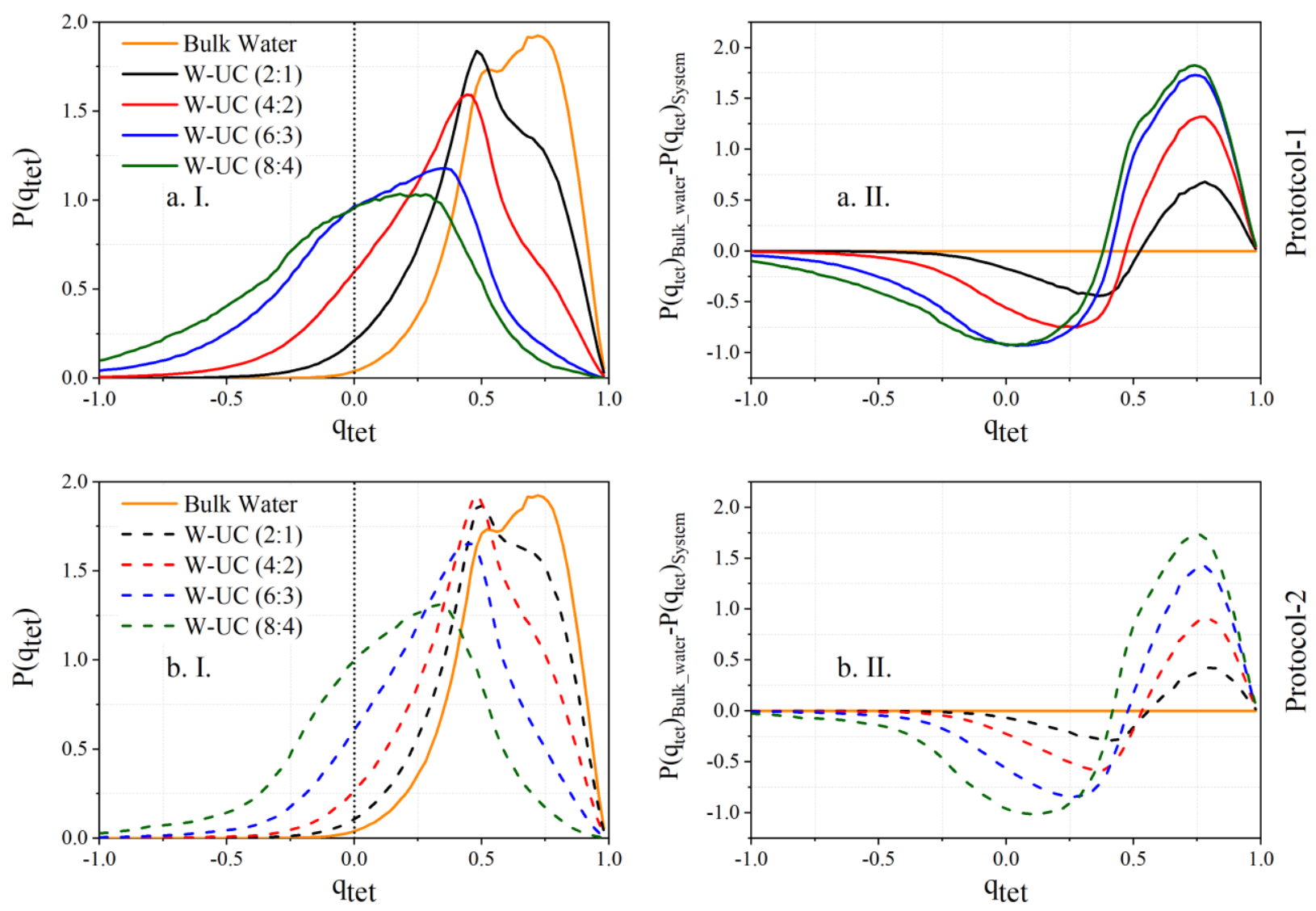

Fig 4: Probability Distributions (P(qtet)) of TOP considering (a.I.) Protocol-1 (solid line), (b.I.) Protocol-2 (dashed line) are plotted in the first column. In the second column, the respective differences of probability distributions of TOP from the probability distribution of TOP for the bulk water are plotted (a.II. \& b.II.).

In the second column of Fig 4, we provide the difference of $P\left(q_{\text {tet }}\right)$ between bulk water and different systems under consideration and the magnitude of its non-zero value is a measure of deviation from the bulk water behavior. In the structured region $\left(q_{t e t}>0.5\right)$, peak height increases as wt $\%$ of reline increases. For this particular plot (Protocol-1 or 2) greater peak height in the structured region signifies a substantial deviation from the tetrahedrality. Conversely, as we move towards a less structured region $\left(q_{\text {tet }}<0.5\right)$, the dip is maximum for W-UC (8:4) system as an increase in reline wt\% shows a greater population in the disordered region. We find a similar trend for Protocol-1 and 2, as can be seen in either of the plots.

The average values of TOP $\left(\left\langle\left(q_{\text {tet }}\right)_{\text {system }}\right\rangle\right)$ calculated for Protocol-1 (solid color bar) and Protocol-2 (striped color bar) are shown in Fig 5 using bar diagrams along with that of the bulk water (solid orange bar). 


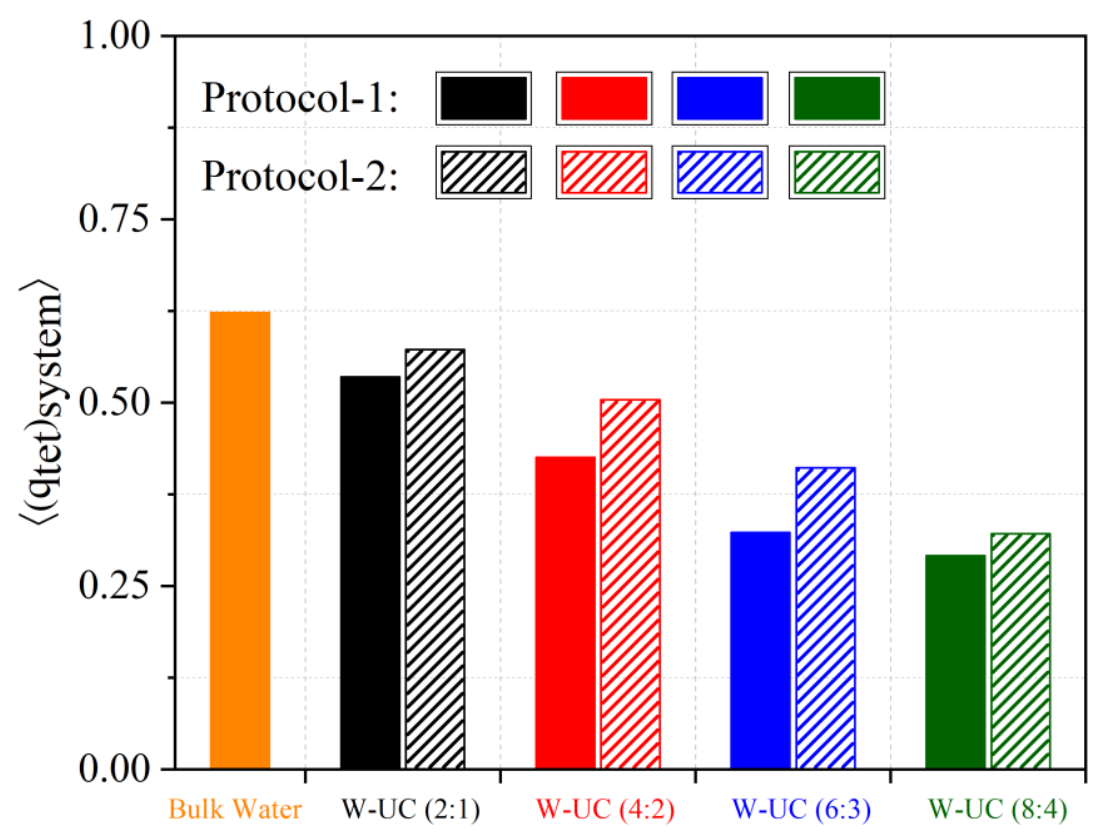

Fig 5: Average values of TOP $\left(\left\langle\left(q_{\text {tet }}\right)_{\text {system }}\right\rangle\right)$ for different systems following Protocol-1 and Protocol-2 are represented as bar plots. For comparison purpose $\left\langle\left(q_{\text {tet }}\right)_{\text {system }}\right\rangle$ for bulk water is also provided.

The summarized outcome of Fig 5 is the gradual decrease in $q_{\text {tet }}$ value as reline wt $\%$ increases in the system. We also encounter an enhanced tetrahedrality in Protocol-2 than Protocol-1 for all the systems under investigation.

\begin{tabular}{|c|c|}
\hline System & \% increase in $\left\langle\left(\boldsymbol{q}_{\text {tet }}\right)_{\text {system }}\right\rangle$ \\
\hline W-UC $(2: 1)$ & 6.95 \\
\hline W-UC $(4: 2)$ & 18.45 \\
\hline W-UC $(6: 3)$ & 27.34 \\
\hline W-UC $(8: 4)$ & 10.37 \\
\hline
\end{tabular}

Table 1: Percentage increase in the value of average TOP $\left\langle\left(q_{\text {tet }}\right)_{\text {system }}\right\rangle$ in Protocol-2 while comparing it with Protocol-1 for different systems.

A comparison between the probability distributions is also provided in the supplementary information (Fig S3) using both the protocols for all the different systems. Table 1 represents the percentage of increment in $\left\langle\left(q_{\text {tet }}\right)_{\text {system }}\right\rangle$ while going from Protocol-1 to Protocol-2. An increasing rise in the enhancement is observed going from W-UC $(2: 1)$ to W-UC (6:3), but the enhancement drops drastically to $~ 10 \%$ from $\sim 27 \%$ from W-UC (6:3) to W-UC (8:4). It can be attributed to the fact that, while accounting for the system W-UC (8:4), the number of water molecules in the system is negligibly small and the environment is highly crowded. Even in the case of Protocol-2, water molecules cannot suitably coordinate with electronegative hetero. This gets reflected in a considerably lesser enhancement of the average TOP value from W-UC (6:3) to W-UC (8:4). 
While calculating $q_{\text {tet }}$ we need to consider the four immediate neighbors of the central water-oxygen ( $\mathrm{Ow}$ ) as partners. Depending upon the protocol of calculation chosen and the concentration of reline in the system, the distance of the neighbors from Ow varies. Fig S4 (supplementary information) represents the probability distribution $(P(s))$ of distance of the partners (according to Protocol-1 and 2) surrounding the central wateroxygen atom $(\mathrm{Ow})$ for different systems investigated and for different neighbors.

\subsubsection{Probability distribution of $\theta_{j k}$}

A closer investigation of $q_{t e t}$ shows that, its value is completely governed by the value of $\theta_{j k}$. Hence the trend we have obtained in Fig 4 can be best described by the distribution of the subtended angle $\left(\theta_{j k}\right)$.
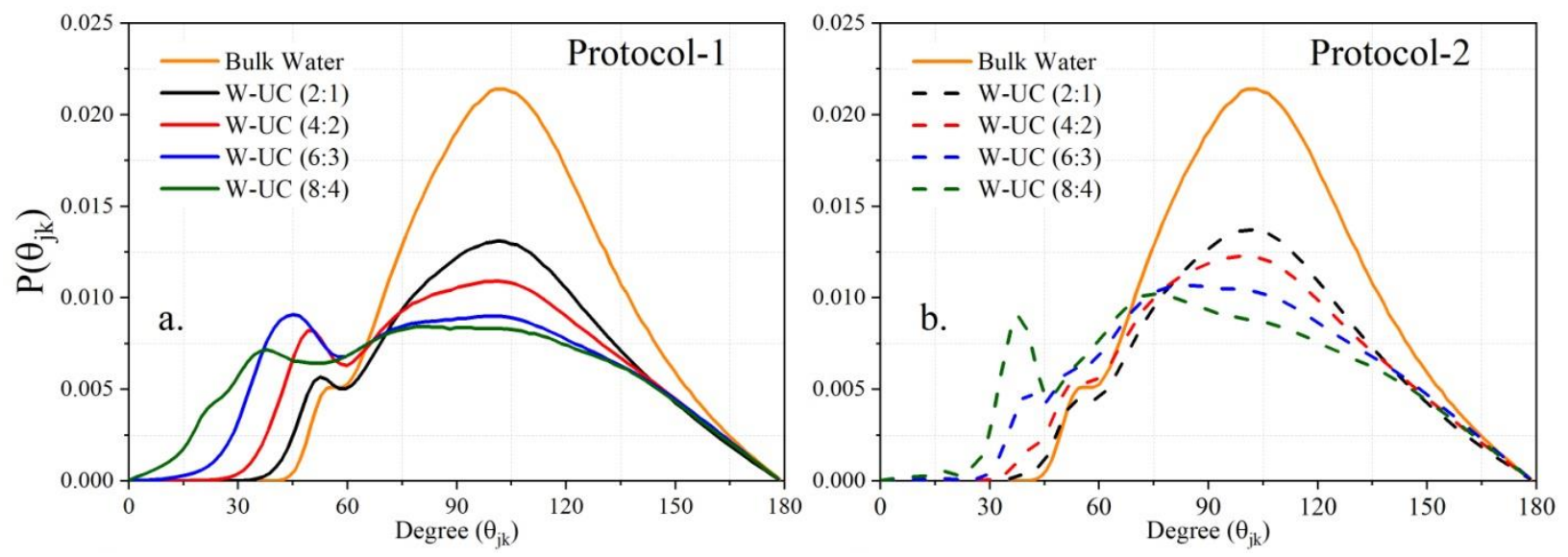

Fig 6: Probability Distribution $\left(\mathrm{P}\left(\theta_{\mathrm{jk}}\right)\right)$ of angle $\theta_{\mathrm{jk}}$ subtended by two partners $(j, k)$ with the central Ow following a. Protocol-1 (solid line representation), b. Protocol-2 (dashed line representation).

In Fig 6, Bulk water is represented in an orange solid line for comparison purposes. It shows that the peak of the probability $\left(P\left(\theta_{j k}\right)\right)$ resides between $100^{\circ}$ and $110^{\circ}$, which accounts for the experimentally observed local tetrahedral angle constituted by the water-oxygen. For Protocol-1 and 2 we see a flattening of the probability plot between $100^{\circ}$ and $110^{\circ}$ and a sharp rise followed by a sudden drop between $30^{\circ}$ and $50^{\circ}$ as the wt\% of reline increases (from W-UC (2:1) to W-UC (8:4)). However, this sharp peak rather resembles a hump when the reline concentration is low. As the reline concentration increases the transformation of a hump to a sharp peak between $30^{\circ}$ and $60^{\circ}$ addresses a noticeable structural distortion of water from tetrahedrality, which is in agreement with an earlier study, ${ }^{68}$ where the addition of urea molecules to the aqueous solution invokes a disturbance in the microscopic structural network of water.

We have already witnessed an increase in average TOP value for Protocol-2 while comparing it with that of the Protocol-1 (Fig 5), for respective systems, this enhancement is originated from the value of the subtended angle $\theta_{j k}$. In Protocol-2 as we have considered electronegative heteroatoms (Nitrogen and 
Oxygen) irrespective of the source, the central Ow finds its partner frequently in a more suitable position that forms an angle which contributes towards sustaining the tetrahedrality. To elucidate this point more clearly, we have plotted $\left(P\left(\theta_{j k}\right)\right)$ following Protocol-1 and Protocol-2 together for all the systems in the supplementary information (Fig S6). Here we find a higher probability of angular values for Protocol-2 in the structured region (between $100^{\circ}$ and $110^{\circ}$ ), whereas the first peak (between $30^{\circ}$ and $60^{\circ}$ ) is much prominent in case of Protocol-1. This trend is consistent in all the systems, which unarguably confirms a recovery of tetrahedral structure considering Protocol-2 but not Protocol-1.

While analyzing Fig 6 for all the systems, we find a co-existence of two humps and the conversion of the first hump to a sharp peak at higher reline concentration. The stability of these two peaks can be expressed in terms of change in free energy and the position of their respective minima. This point out the propensity of a system to move from the highly structured region to the locally stable unstructured region and viceversa. The change in free energy $(\Delta G)$ is calculated as follows,

$$
\Delta G=-R T \ln P\left(\theta_{j k}\right)
$$

The free energy plots correspond to the subtended angle distribution are provided in the supplementary information (Fig S7) using the same color code as in Fig 6.

\subsubsection{Contribution of the cosolvent and recovery of tetrahedrality}

As evident from the earlier plots and calculations, we quantify the recovery of tetrahedrality in terms of orientational tetrahedral order parameter in Protocol-2, which is mainly driven by the subtended angle $\theta_{j k}$ as evident from Fig S6 in the supplementary information.

The structural recovery observed in Fig 5 and Table 1 is due to the frequent presence of the co-solvents as the immediate neighbors. In Fig 7 we represent the contribution of the water, urea, and choline cation towards $q_{\text {tet }}$ at different neighboring positions for which a substantial increment in $q_{\text {tet }}$ is observed in Protocol-2. The fraction of contribution as a partner while calculating $q_{t e t}$ is designated by $\gamma_{C}$ in Fig 7 . 

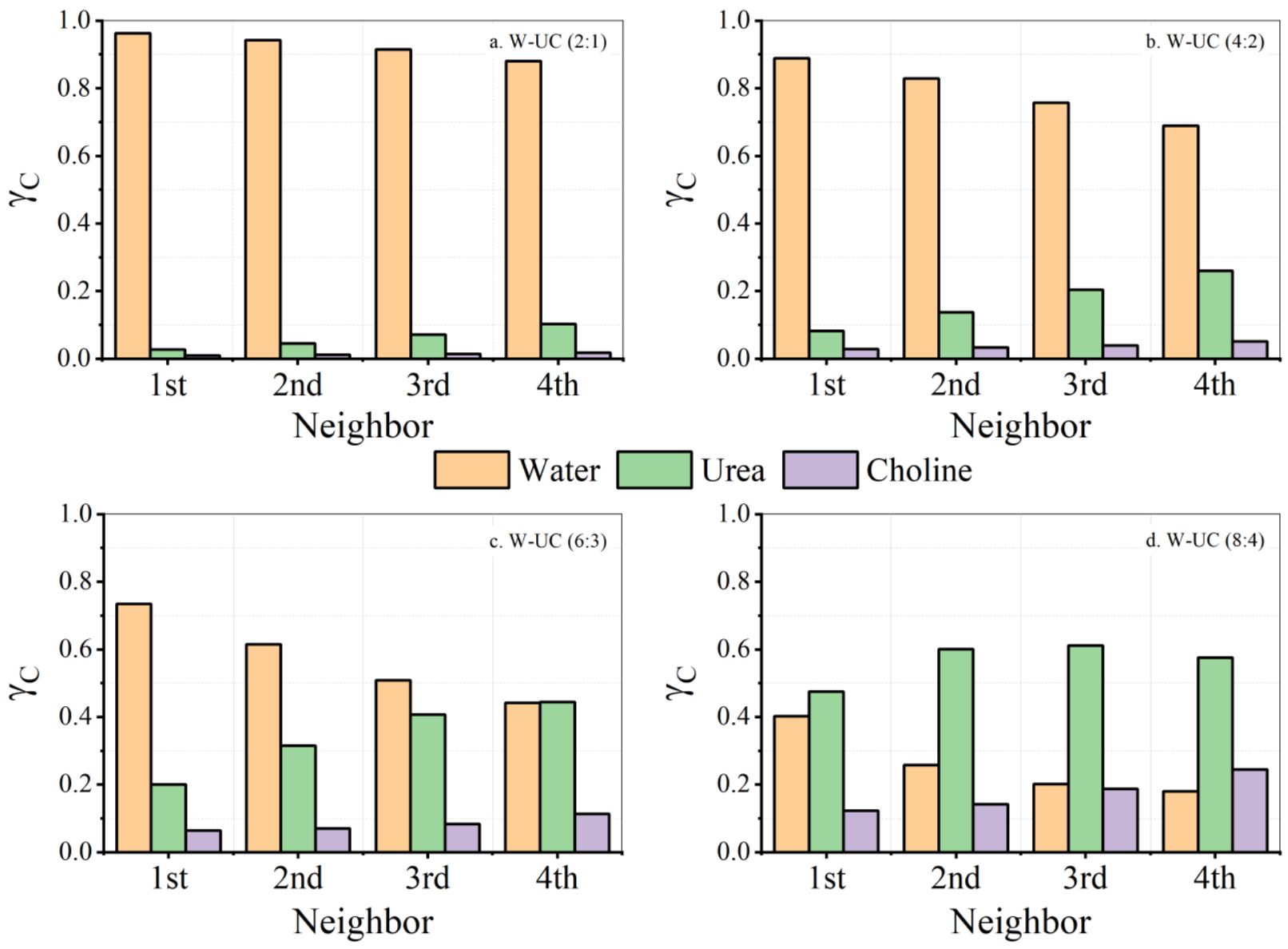

Fig 7: Contribution of the constituents in calculating $q_{\text {tet }}$ as four immediate neighbors in the systems considered for this investigation using Protocol-2.

It is evident from Fig 7 that in case of the W-UC (2:1) system, the neighbors are majorly populated by the water molecules having a value of $\gamma_{C} \sim 0.9$ and the presence of the other constituents as neighboring partners is negligibly small. The presence of water molecules as neighbors decreases as the wt $\%$ of reline increases which causes the increment in $q_{\text {tet }}$ in Protocol-2. As can be seen from Fig 7, for every system we find a steady decrease in contribution from the water molecules while considering the fourth neighbor, hence accountable numbers of urea and choline cation are populated in the fourth neighboring position even when the wt\% of reline is low enough. Therefore, the bulging observed in Fig S3 (supplementary information) majorly comes from the consideration of co-solvents as the fourth position. In W-UC (4:2) system, almost $20 \%$ contribution at the fourth neighboring position comes from urea which even increases further in W-UC (6:3) and equally shares $80 \%$ of the total partner contribution with water. We further increase the wt $\%$ of reline to $\sim 91 \%$ in the case of W-UC (8:4), where urea and choline cation contributes almost $60 \%$ to $70 \%$ of the total partner count at all neighbors. Fig 7 provides an idea that even in the low reline concentration particularly the fourth neighboring position started contributing to TOP that makes a 
difference in Protocol-1 and 2 and this neighbor contribution keeps on increasing and contributes heavily to further addition of reline along with other neighbors.

\subsection{Analysis of Hydrogen bonded solution structure}

\subsubsection{Substitution of number of hydrogen bonds}

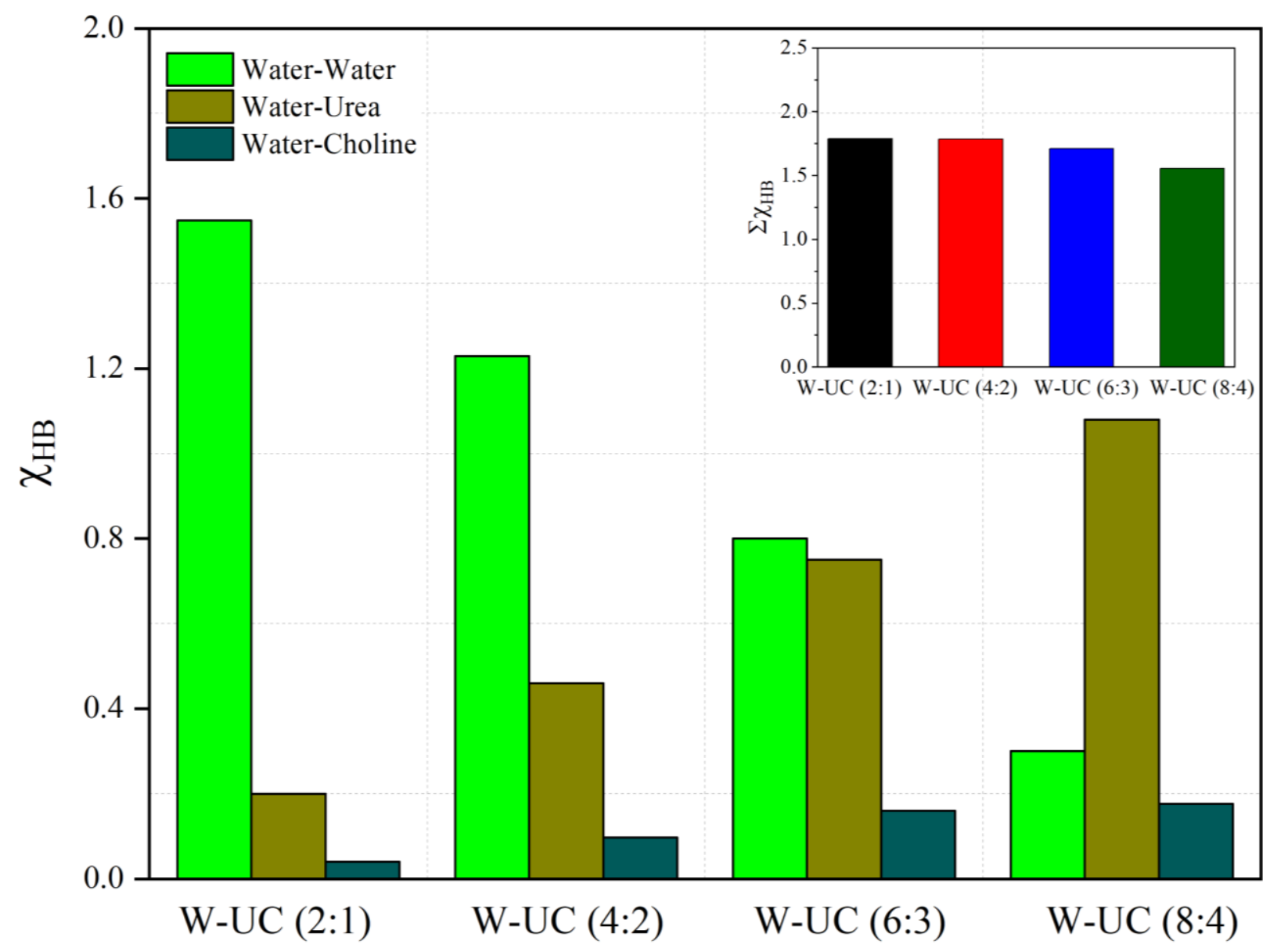

Fig 8: Bar plot representation of scaled hydrogen bond number $\left(\chi_{H B}\right)$ for water-water, water-urea, water-choline for different systems under consideration. In the inset, the sums of $\chi_{H B} \mathrm{~s}$ of all three aforesaid pairs are provided.

We further analyze the number of hydrogen bonds between different constituents in the system. For this, we have considered a parameter $\chi_{H B}$ to account for the number of hydrogen bonds formed between water molecules and other co-solvents and we termed it as the scaled hydrogen bond number $\chi_{H B}$ and defined it as follows,

$$
\chi_{H B}=\frac{n_{H B}}{n_{H_{2} O}}
$$


Here, $n_{H B}$ denotes the number of hydrogen bonds between two specific constituents, such as water-water, water-urea, water-choline and $n_{\mathrm{H}_{2} \mathrm{O}}$ is the number of water molecules in that particular pair.

Fig 8 depicts that, as reline wt $\%$ increases there is a decrease in $\chi_{H B}$ (Water-Water), and a simultaneous increase in $\chi_{H B}$ (Water-Urea) and $\chi_{H B}$ (Water-Choline). This observation is quite expected as the number of urea and choline molecules increases with increase in wt $\%$ of reline. It is also evident from Fig 8 that the water-water hydrogen bond is substantially replaced by water-urea hydrogen bonds. A drastic reduction of $\sim 75 \%$ in the magnitude of $\chi_{H B}$ (Water-Water) is observed while comparing W-UC (8:4) and W-UC (2:1). Simultaneously $\chi_{H B}$ (Water-Urea) increases five folds in W-UC (8:4) compared to W-UC (2:1) and dominantly water-urea hydrogen bonds govern the hydrogen bond network in the system. $\chi_{H B}$ (WaterWater) and $\chi_{H B}$ (Water-Urea) shows comparable values in W-UC (6:3) system, and then $\chi_{H B}$ (Water-Urea) surpasses $\chi_{H B}$ (Water-Water) on further addition of co-solvents into the system.

In the inset of Fig 8 , we have plotted $\sum \chi_{H B}$ which is calculated as follows,

$$
\sum \chi_{H B}=\frac{n_{H B}(\text { Water }- \text { Water })+n_{H B}(\text { Water }- \text { Urea })+n_{H B}(\text { Water }- \text { Choline })}{n_{\mathrm{H}_{2} \mathrm{O}}}
$$

We find constancy in $\sum \chi_{\boldsymbol{H} \boldsymbol{B}}$ value for all systems under consideration, though a little reduction in W-UC (8:4) system is observed, which is due to a stark reduction in the number of water-water hydrogen bonds. This constancy in the number of hydrogen bonds while adding co-solvents is in agreement with that of the molecular dynamics study by Stumpe et al. ${ }^{99}$

In the supplementary information (Fig S8) we have provided the numbers of hydrogen bonds between choline and urea per choline cations. We find that with an increase in the reline wt $\%$ in the system the number of hydrogen bonds increases from 0.1 (in W-UC (2:1)) to 0.7 (in W-UC (8:4)). It accounts for the closer proximity and stronger interaction between choline and urea at higher concentrations.

\subsubsection{Microscopic origin of structural recovery}

While analyzing the water structure, we find that different water molecules are surrounded by different numbers of co-solvents, along with other water molecules. The successful numbers of co-ordination of a central water molecule are calculated using the geometric criteria of hydrogen bonding. ${ }^{100}$ We have taken all donor-acceptor possibilities into account that could potentially form a hydrogen bond. Different modes of hydrogen bond formation for water molecules are provided in the supplementary information for a better understanding (Table S3). 


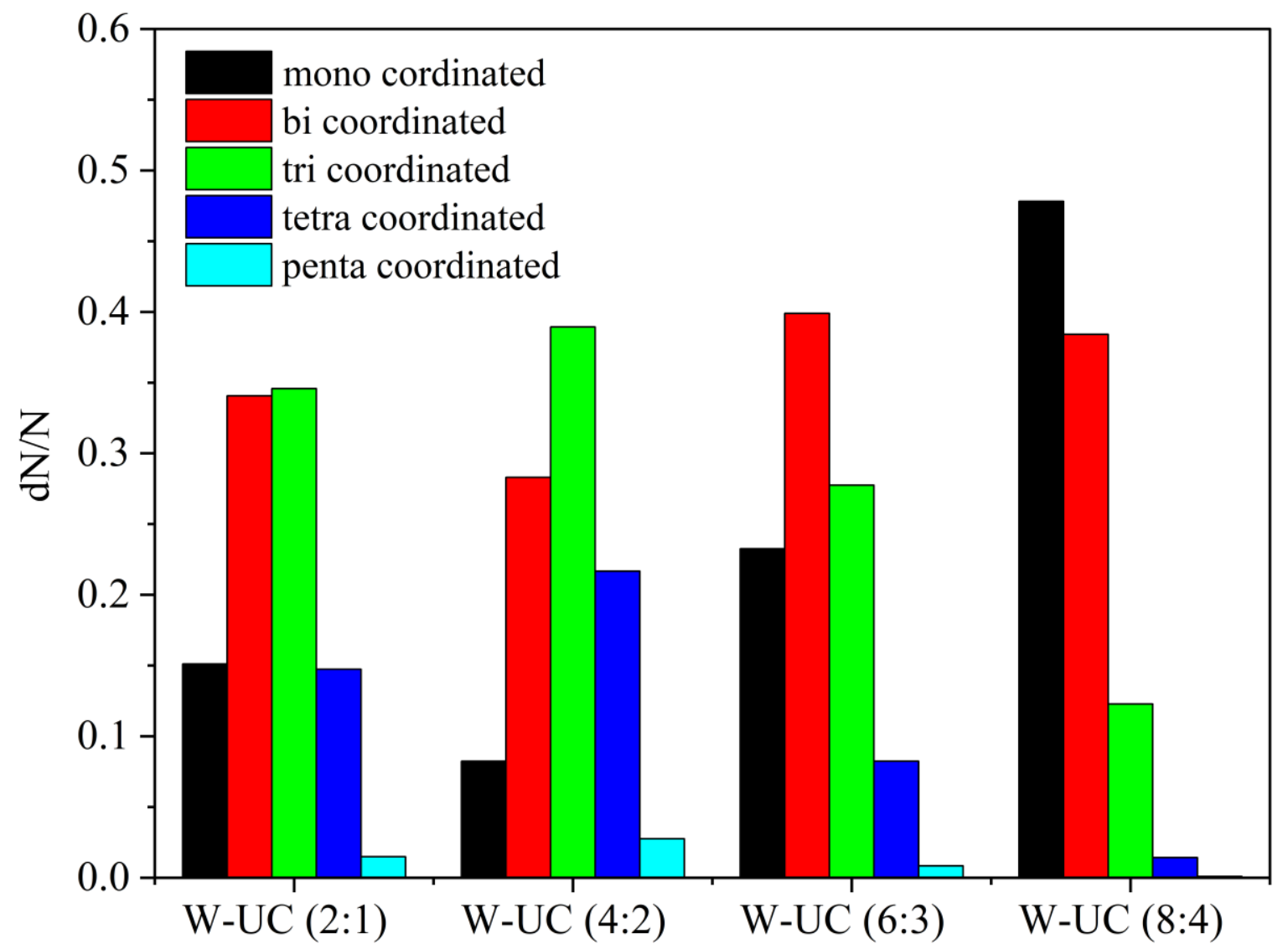

Fig 9: Fraction of mono, bi, tri, tetra, and penta coordinated successful hydrogen bonds with a central water molecule for different systems considering all constituents as potential donor/acceptor having proper hydrogen bond-forming site.

As can be seen from Fig 9, the bi and tri coordinated (red and green bar respectively) water molecules play the major role in deciding the hydrogen-bonded structure, but in the case of W-UC (8:4), which is the densest system considered, an abundance of mono coordinated (black bar) central water molecules was found that govern almost $50 \%$ of the total hydrogen-bonded structure. Calculation of TOP considering Protocol-1 and 2 (Fig S3, green solid line and dashed line) evaluates this particular system to be the most disordered one, which is the consequence of the major absence of multiple successful hydrogen bonds especially the simultaneous formation of four successful hydrogen bonds, evident from Fig 9. For other systems, we observe around $70 \%$ of the central water molecule is either bi or tri coordinated. The presence of water molecules with lower coordination numbers has been confirmed using dielectric relaxation spectroscopy. For example, Lu et al. estimated the average hydrogen bond number per water from the activation enthalpy obtained from dielectric relaxation spectroscopy of DMSO solutions. ${ }^{101}$ The numbers vary between 2 and 3 justifying the presence of bi or tri coordinated water molecules. Similar results were found when the effective hydration number of choline was monitored in aqueous choline chloride solutions 
by Shaukat et al. ${ }^{102}$ The average hydrogen bond numbers in the ternary mixtures considered in the present study are influenced by both choline and urea molecules and a properly designed similar experiment, on the mixtures with higher reline wt $\%$ can in principle confirm the presence of bi and tri coordinated water molecules. The formation of a proper tetrahedral water structure requires four hydrogen-bonded partners but it is evident that successful tetra coordination (blue bar) is maximum in the case of W-UC (4:2) which is $20 \%$ of the total hydrogen-bonded network. The value of the contribution of tetra-coordinated water molecules for the W-UC (8:4) system is less than $5 \%$ and as a result, the microscopic water structure deviates severely from tetrahedrality. In Fig 9, we have also considered the penta coordinated (sky blue bar) central water molecules but it shows a negligibly small contribution, and therefore not considered here. Further, the numbers of different constituents connected to water with a specific coordination number have been calculated. Based on these constituent numbers, the coordination number has been classified into different binding modes and represented as triplet of numbers consisting of the numbers of Water (W), Urea (U), and Choline (C) coordinated to it. The triplet of numbers used in the next few plots (Fig 10 a-d) designates successful coordination of a central water molecule by water (W), urea (U), and choline (C) respectively as it is provided in Table S3 in the supplementary information. We use the term coordination to simply explain the number of successful hydrogen bonds with the central water molecule. We provide the representative structure of the majorly contributing binding modes in the following plots. 


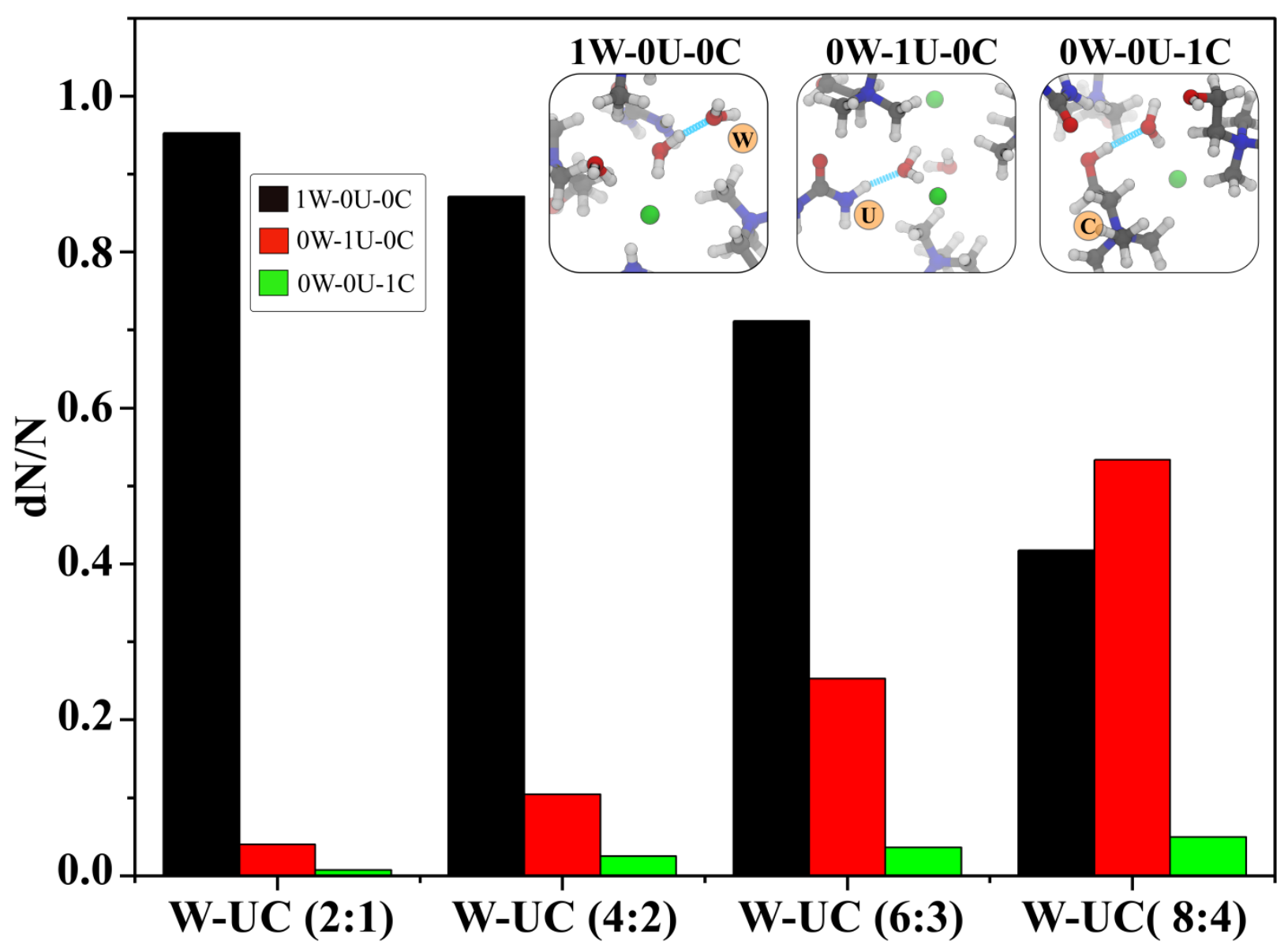

Fig 10a: Fraction of different possible mono coordinated binding modes for successful hydrogen bond formation with a central water molecule for different systems considering all constituents as potential donor/acceptor with proper hydrogen bond-forming site. In the plot, we represent the diagram of the major contributing modes only.

Fig 10a explains the different coordination possibilities of mono coordinated water molecules. In W-UC (2:1) system where reline wt $\%$ is only $26 \%$, we find above $95 \%$ of the mono coordinated (Fig 10a, black bar) water molecules are coordinated only with water molecules. The presence of urea and choline in the coordination sphere is negligible. As reline wt\% increases the potential contribution in the mono coordination mode is coming from the urea molecules and it reaches to cover maximum of $50 \%$ of the mono coordinated (Fig 10a, red bar) water molecules which are singly and successfully hydrogen-bonded by a urea molecule in the W-UC (8:4) system. The contribution of singly hydrogen-bonded water molecules by a water molecule reduces to $40 \%$ of the total mono coordinated counts (Fig 10a, black bar). This clearly shows the proper positioning of a urea molecule near the central water molecule that could potentially form the hydrogen bond. 


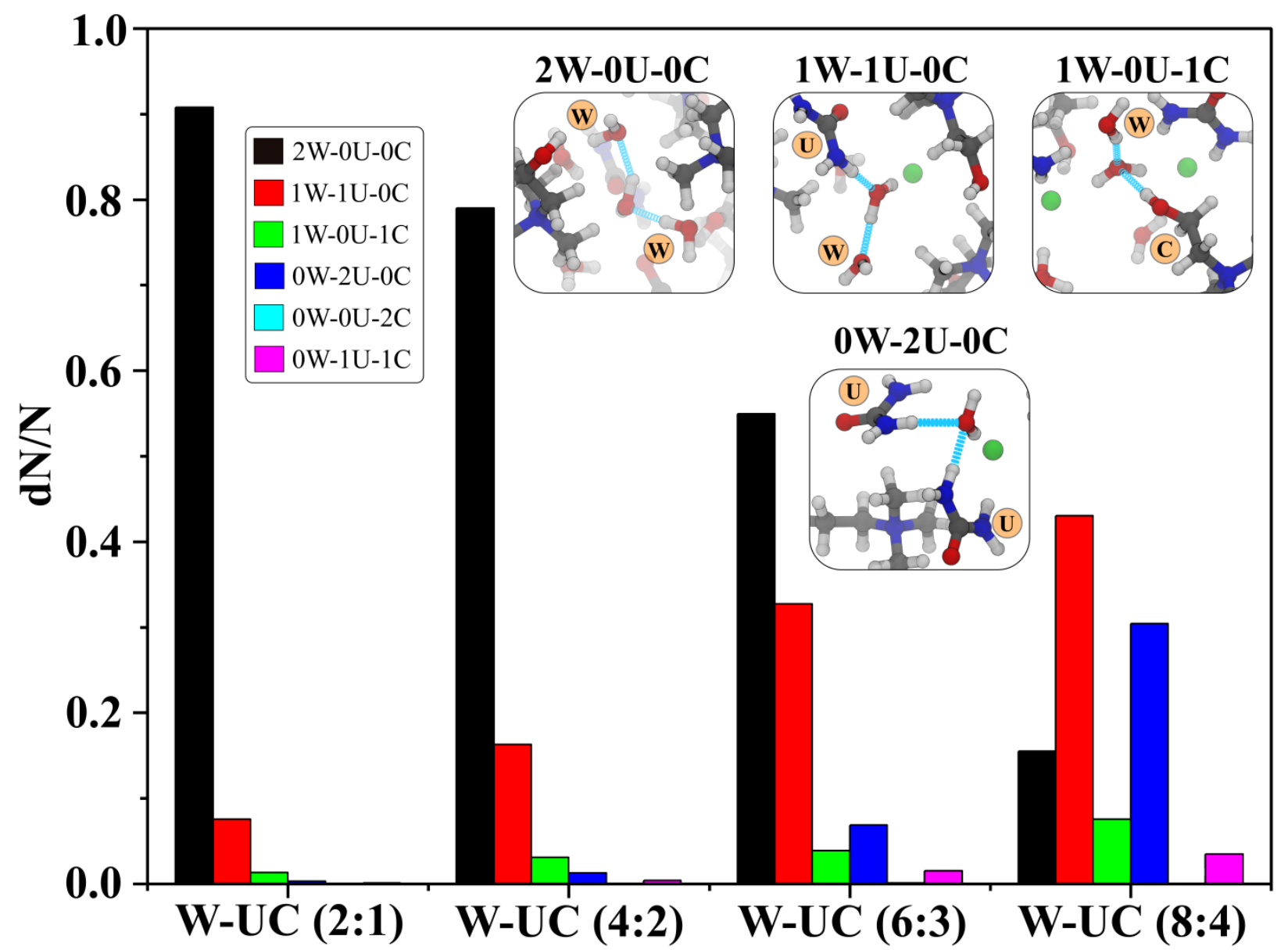

Fig 10b: Fraction of different possible bi coordinated binding modes for successful hydrogen bond formation with a central water molecule for different systems considering all constituents as potential donor/acceptor having proper hydrogen bond-forming site. In the plot, we represent the diagram of the major contributing modes only.

Fig 10b sheds light on the bi coordinated water molecules. We find that for W-UC (2:1), W-UC (4:2), and W-UC (6:3) the bi coordination mode entirely comes from the water molecule (Fig 10b, black bar) and it is around 95\%,80\%, 55\% respectively. Other modes of bi coordination contribute negligibly in the aforesaid systems but an increase in the number of co-solvents in the system favors the coordination from the urea molecules and one of the water molecules is successfully replaced by one of the urea molecules in the major contributing mode within the coordination sphere (Fig 10b, black bar height gradually decreases and red bar height gradually increases as reline wt\% increases). A closer look into the W-UC (6:3) system depicts the simultaneous coordination from the water and urea (Fig 10b, red bar) contributes around 30\% of total bi coordination. In W-UC (8:4) we find a substantial amount of water molecules where bi coordination entirely comes from the urea (Fig 10b, blue bar) which covers $\sim 30 \%$ of the total bi coordinating binding mode. Also, in W-UC (8:4), simultaneous coordination from urea and water (Fig 10b, red bar) contributes $\sim 40 \%$ of the binding mode which is maximum in this system. 


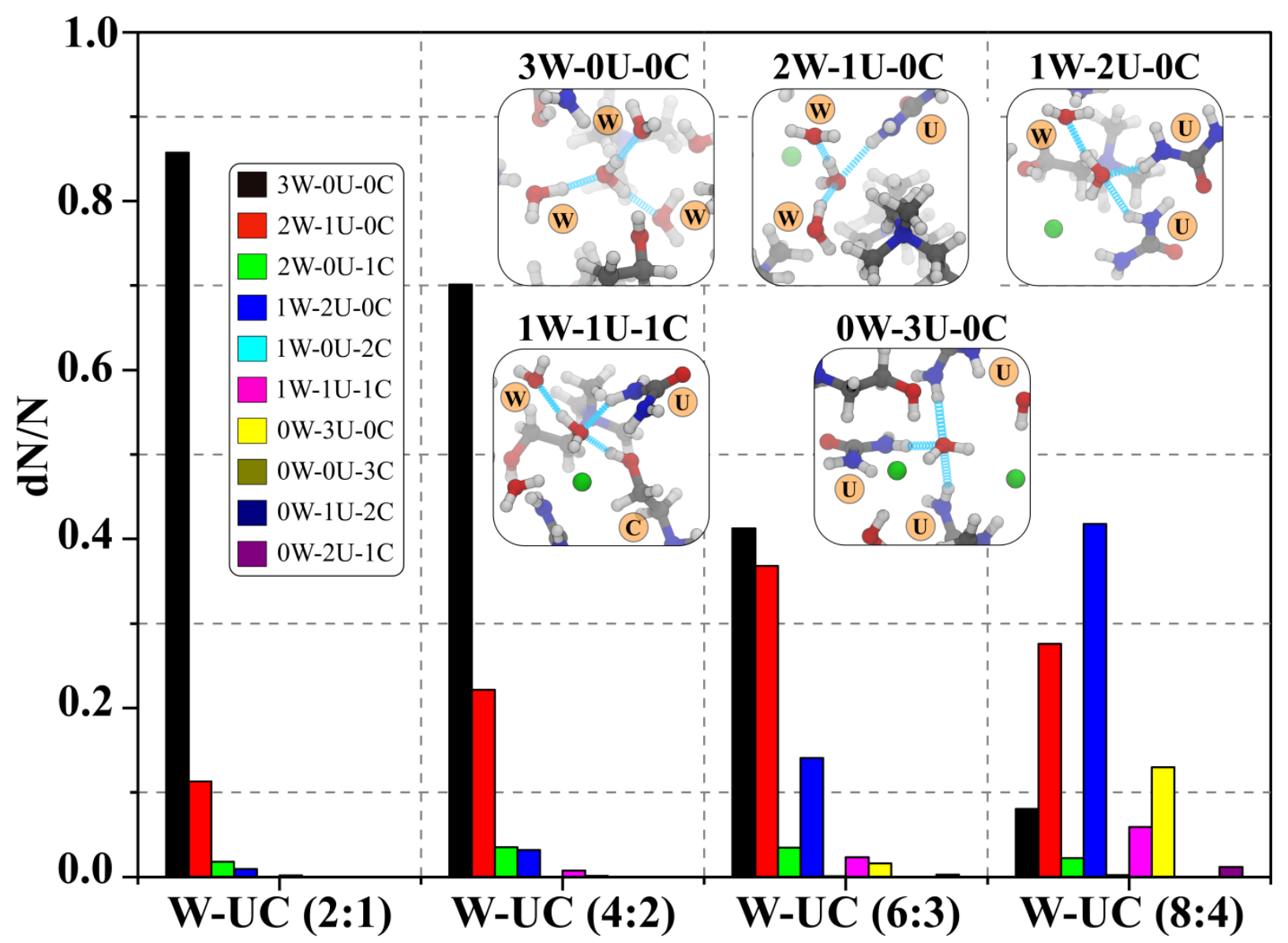

Fig 10c: Fraction of different possible tri coordinated binding modes for successful hydrogen bond formation with a central water molecule for different systems considering all constituents as potential donor/acceptor having proper hydrogen bond-forming site. In the plot, we represent the diagram of the major contributing modes only.

It is evident from Fig 10c that coordination by water molecule is pivotal for W-UC (2:1), W-UC (4:2), and W-UC (6:3) (Fig 10c, black bar). This observation is similar to that of the mono and bi coordinated (black bar in Fig 10a and Fig 10b) water molecules where we find a potential contribution coming from the occupancy of space only by the water molecules. An increase in reline wt $\%$ increases the coordination possibility by urea and choline which is very likely. The direct and most prominent outcome of the inclusion of co-solvent is the replacement of a water molecule by a urea molecule within the coordination sphere (Fig 10c, decrease in the height of the black bar and rise in the height of the red, blue, yellow bars). As we include urea and choline into the systems, W-UC (4:2) system onwards, at least one of the coordination sites always remains occupied by the atoms from urea molecules (Fig 10c, red bar), while in W-UC (8:4) system tri coordinated binding mode that contains two atoms from the urea molecules contributes maximum (Fig 10c, blue bar). Another astonishing observation is the gradual rise of the yellow bar which signifies that the central water molecule could even accommodate three potential hydrogen bond-forming atoms from the urea molecules. 


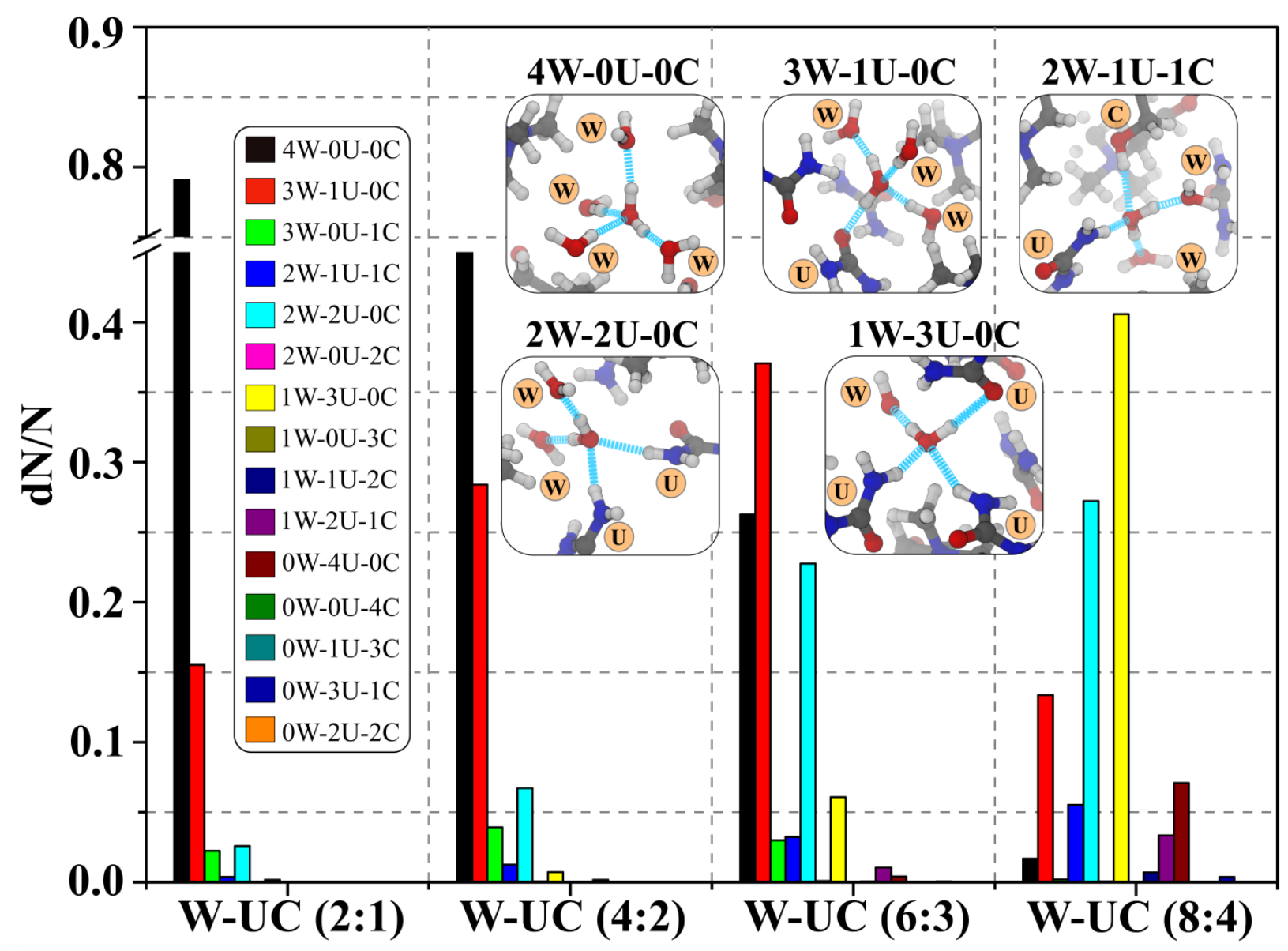

Fig 10d: Fraction of different possible tetra coordinated binding modes for successful hydrogen bond formation with a central water molecule for different systems considering all constituents as potential donor/acceptor having proper hydrogen bond-forming site. In the plot, we represent the diagram of the major contributing modes only.

Fig $10 \mathrm{~d}$ also shows that even in the case of tetra coordinated binding mode for lower reline concentration, successful hydrogen bond is only formed by the water molecules (Fig 10d, black bar). Similar to the tri coordinated water molecules hydrogen bond formation by urea molecules increases (red, sky blue, and yellow bar) as reline wt\% increases. Though the percentage of water molecules that undergo successful tetra coordination is negligible for W-UC (8:4) as evident from Fig 9, the presence of three coordination sites (yellow bar) and four coordination sites (brown bar) entirely contributed from urea molecule is not negligible.

\section{Conclusion}

We use extensive all-atom molecular dynamics simulations to explore the microscopic characteristics of water in the presence of co-solvents. In this investigation, we use reline as the co-solvent in a solution which consists of a well-known protein denaturant urea and a well-known protein secondary structure protecting 
osmolyte choline chloride. We gradually increase the amount of reline within the system to observe the change it could invoke around the water. An accumulation of water molecules is observed while the wt $\%$ of reline is increased in the system, as evident from the radial distribution plot. There is also the likelihood of more amounts of co-solvents surrounding the water molecules (Fig 2). The position of the water-oxygen (Ow) self pair-correlation peak is in good agreement with that of the experimentally verified one ${ }^{103}$. Further insights are drawn from the relative number fraction $\left(\Phi_{i}(r)\right.$ ), which accounts for the spatial occupancy of the constituents surrounding the water-oxygen $(\mathrm{Ow})$. It shows whenever there is an increase in the amount of water surrounding the water molecule there is an overall decrease in the amount of urea and choline (Fig 3).

The internal structural arrangement of water is further explored by computing the orientational order parameter $\left(q_{\text {tet }}\right)$, the value of which varies between 0 and 1 . The value 0 signifies complete random orientation, while the value 1 confirms the ice-like tetrahedral structure. In our work, we witness a gradual deviation from tetrahedrality as we introduce reline in water and increases reline concentration (Fig 4). Consideration of Protocol-2 shows an increase in the tetrahedrality of the water molecules as the central water molecule has more partners (Fig 5). But this increased in tetrahedrality in Protocol-2 compared to Protocol-1 is non-monotonic (Table 1). As discussed earlier the value of $q_{t e t}$ is dependent on the subtended angle $\theta_{j k}$ and we find a stabilization value of $\sim 1 \mathrm{kcal} / \mathrm{mol}$ by converting this to a free energy landscape that looks like a double well. This signifies structural disorder for bulk water, but this difference decreases gradually as the amount of reline increases in the system (Fig S7, supplementary information). However, this stabilization is negligible as it is comparable to the thermal fluctuations.

The water internal structure is guided by the enhanced hydrogen bond network, and we have quantified it by calculating the scaled hydrogen bond number $\left(\chi_{H B}\right)$. It reveals the substitution of water-water hydrogen bonds substantially by the water-urea hydrogen bonds and to some extent by the water-choline hydrogen bonds. Interestingly choline has significant hydrogen-bonded interactions with urea molecules. A plot of the number of urea-choline hydrogen bonds in different systems indicates an enhanced urea-choline interaction with increased reline concentration (Fig S8, supplementary information). The important role of choline mediated hydrogen bonds in reline and other deep eutectic mixtures has been shown by Minofar and group. ${ }^{104-106}$ However we focused to elucidate the structure of water in the presence of reline with varying concentrations. We strengthen our study by calculating the fraction of water molecules that could effectively accommodate different numbers of hydrogen bonds. We find that while the wt\% of water is higher the system is mainly driven by the bi and tri coordinated water molecules, an increase in reline wt $\%$, especially in case of W-UC (8:4) system is driven by the mono coordinated water molecules. Further analyses of the different modes of binding for mono, bi, tri, tetra coordinated water molecules reveal that 
the successful hydrogen bonds made by water molecules with other water molecules are gradually replaced by urea those enter the coordination sphere of the water molecules. For example, in the case of tetra coordinated water molecules, we find a gradual replacement of the water-bound sites by the urea hydrogen bond sites, as can be seen from the bar plot diagrams. From these aforesaid plots (Fig 10 a-d) it is evident that owing to the presence of multiple potential hydrogen bonding sites the scenario of water structure recovery in terms of tetrahedrality mainly comes from the urea molecules as it actively penetrates the water coordination sphere whereas the bulky choline molecules do not significantly participate in hydrogen bond formation. This in turn accounts for the interference of urea molecules within the hydrogen-bonded water molecules. Hence it proves the crucial role played by the hydrogen bond network in deciding the overall property of a system. We believe now we have a better understanding of the change in the microscopic picture of water structure in the presence of reline as the latter is varied from low to high concentration. The reline and aqueous reline solution have important roles to modulate the solvation and stabilization of large macromolecules like protein, nucleic acid, polymer, etc. For example, (i) aqueous reline solution can prevent the unfolding of HP-36 by urea, ${ }^{40}$ (ii) it can stabilize the quartet DNA conformation ${ }^{107}$ and (iii) it also helps in the solvation of PEG $400^{105}$. The understanding of the role of hydrogen bond acceptor and donor in deep eutectic solvent (DES) from this study can motivate the design of more efficient DES for specific purposes by modulating the chemical nature of the constituents and their compositions.

\section{Supplementary Information}

A brief overview of the simulated systems; Comparison between experimental and simulated density; RDF of the constituents for all systems; Comparison of $\mathrm{P}\left(q_{t e t}\right)$ between Protocol-1 \& 2; Distance distribution of the four immediate neighbors; Probability distribution of the subtended angle for Protocol-1 and 2 and free energy representation; Number of hydrogen bonds between urea and choline; Hydrogen bond coordination statistics.

\section{Author Information}

\section{Corresponding Author}

"Email: rajarshi@chem.iitb.ac.in

Mailing Address: Department of Chemistry, Indian Institute of Technology Bombay, Powai, Mumbai400076, India.

Phone: + 91-022-2576 7192.

Fax: + 91-022-2576 7152.

\section{Disclosure Statement}

The authors declare no competing financial interest. 


\section{Acknowledgment}

R.C. acknowledges SERB (Grant SB/SI/PC-55/2013) for financial support. S.S. and A.M. thanks IIT Bombay for fellowship. Authors cordially thank Subhasish Chaki, Praveen Kumar, Asha Rani Chaudhuri for technical help and R. Kailasham for critical reading of the manuscript. We dedicate this work to Prof. K. L. Sebastian for his mentorship and encouragement over the years.

\section{Keywords}

Choline chloride, Urea, Water structure, Tetrahedral order parameter, Hydrogen bond

\section{References}

1 A. P. Abbott, R. C. Harris and K. S. Ryder, Application of hole theory to define ionic liquids by their transport properties, J. Phys. Chem. B, 2007, 111, 4910-4913.

2 S. L. Waite, H. Li and A. J. Page, NO2 Solvation Structure in Choline Chloride Deep Eutectic Solvents - The Role of the Hydrogen Bond Donor, $J$. Phys. Chem. B, 2018, 122, 4336-4344.

3 A. Faraone, D. V. Wagle, G. A. Baker, E. C. Novak, M. Ohl, D. Reuter, P. Lunkenheimer, A. Loidl and E. Mamontov, Glycerol Hydrogen-Bonding Network Dominates Structure and Collective Dynamics in a Deep Eutectic Solvent, J. Phys. Chem. B, 2018, 122, 1261-1267.

4 B. Singh, H. Lobo and G. Shankarling, Selective N-alkylation of aromatic primary amines catalyzed by bio-catalyst or deep eutectic solvent, Catal. Letters, 2011, 141, 178-182.

5 C. Li, D. Li, S. Zou, Z. Li, J. Yin, A. Wang, Y. Cui, Z. Yao and Q. Zhao, Extraction desulfurization process of fuels with ammonium-based deep eutectic solvents, Green Chem., 2013, 15, 2793-2799.

6 A. Das and R. Biswas, Dynamic Solvent Control of a Reaction in Ionic Deep Eutectic Solvents: Time-Resolved Fluorescence Measurements of Reactive and Nonreactive Dynamics in (Choline Chloride + Urea) Melts, J. Phys. Chem. B, 2015, 119, 10102-10113.

7 A. P. Abbott, G. Capper, K. J. McKenzie and K. S. Ryder, Electrodeposition of zinc-tin alloys from deep eutectic solvents based on choline chloride, $J$. Electroanal. Chem., 2007, 599, 288-294. 
8 A. P. Abbott, T. Z. Abolibda, W. Qu, W. R. Wise and L. A. Wright, Thermoplastic starch-polyethylene blends homogenised using deep eutectic solvents, $R S C A d v$., 2017, 7, 7268-7273.

9 J. H. Liao, P. C. Wu and Y. H. Bai, Eutectic mixture of choline chloride/urea as a green solvent in synthesis of a coordination polymer: $[\mathrm{Zn}(\mathrm{O} 3 \mathrm{PCH} 2 \mathrm{CO}$ 2)]·NH4, Inorg. Chem. Commun., 2005, 8, 390-392.

10 S. M. Wang, W. L. Chen, E. B. Wang, Y. G. Li, X. J. Feng and L. Liu, Three new polyoxometalate-based hybrids prepared from choline chloride/urea deep eutectic mixture at room temperature, Inorg. Chem. Commun., 2010, 13, 972-975.

11 G. García, S. Aparicio, R. Ullah and M. Atilhan, Deep eutectic solvents: Physicochemical properties and gas separation applications, Energy and Fuels, 2015, 29, 2616-2644.

12 F. S. Oliveira, A. B. Pereiro, L. P. N. Rebelo and I. M. Marrucho, Deep eutectic solvents as extraction media for azeotropic mixtures, Green Chem., 2013, 15, 1326-1330.

13 J. D. Gamarra, K. Marcoen, A. Hubin and T. Hauffman, Electrode-electrolyte interactions in choline chloride ethylene glycol based solvents and their effect on the electrodeposition of iron, Electrochim. Acta, 2019, 312, 303312.

14 Y. Oh, S. Park, E. Yoo, S. Jo, J. Hong, H. J. Kim, K. J. Kim, K. K. Oh and S. $\mathrm{H}$. Lee, Dihydrogen-bonding deep eutectic solvents as reaction media for lipase-catalyzed transesterification, Biochem. Eng. J., 2019, 142, 34-40.

15 R. Patel, M. Kumari and A. B. Khan, Appl. Biochem. Biotechnol., 2014, 172, 3701-3720.

16 T. Vasantha, P. Attri, P. Venkatesu and R. S. R. Devi, Structural basis for the enhanced stability of protein model compounds and peptide backbone unit in ammonium ionic liquids, J. Phys. Chem. B, 2012, 116, 11968-11978.

17 A. P. Abbott, D. Boothby, G. Capper, D. L. Davies and R. K. Rasheed, Deep Eutectic Solvents formed between choline chloride and carboxylic acids: Versatile alternatives to ionic liquids, J. Am. Chem. Soc., 2004, 126, 9142 9147.

18 S. Kaur and H. K. Kashyap, Unusual Temperature Dependence of Nanoscale Structural Organization in Deep Eutectic Solvents, J. Phys. Chem. B, 2018, 
122, 5242-5250.

19 O. S. Hammond, D. T. Bowron and K. J. Edler, Liquid structure of the choline chloride-urea deep eutectic solvent (reline) from neutron diffraction and atomistic modelling, Green Chem., 2016, 18, 2736-2744.

20 S. Kaur, A. Gupta and H. K. Kashyap, Nanoscale Spatial Heterogeneity in Deep Eutectic Solvents, J. Phys. Chem. B, 2016, 120, 6712-6720.

21 H. K. Kashyap, C. S. Santos, H. V. R. Annapureddy, N. S. Murthy, C. J. Margulis and E. W. Castner, Temperature-dependent structure of ionic liquids: X-ray scattering and simulations, Faraday Discuss., 2012, 154, 133143.

22 H. K. Kashyap, C. S. Santos, N. S. Murthy, J. J. Hettige, K. Kerr, S. Ramati, J. Gwon, M. Gohdo, S. I. Lall-Ramnarine, J. F. Wishart, C. J. Margulis and E. W. Castner, Structure of 1-alkyl-1-methylpyrrolidinium bis(trifluoromethylsulfonyl) amide ionic liquids with linear, branched, and cyclic alkyl groups, J. Phys. Chem. B, 2013, 117, 15328-15337.

23 H. V. R. Annapureddy, H. K. Kashyap, P. M. De Biase and C. J. Margulis, J. Phys. Chem. B, 2011, 115, 9507-9508.

24 H. K. Kashyap and C. J. Margulis, (Keynote) Theoretical Deconstruction of the X-ray Structure Function Exposes Polarity Alternations in Room Temperature Ionic Liquids, ECS Trans., 2013, 50, 301-307.

25 S. Sharma, A. Gupta, D. Dhabal and H. K. Kashyap, Pressure-dependent morphology of trihexyl(tetradecyl)phosphonium ionic liquids: A molecular dynamics study, J. Chem. Phys., 2016, 145, 134506.

26 D. Bandyopadhyay, Y. Kamble and N. Choudhury, How Different Are the Characteristics of Aqueous Solutions of tert-Butyl Alcohol and Trimethylamine-N-Oxide? A Molecular Dynamics Simulation Study, $J$. Phys. Chem. B, 2018, 122, 8220-8232.

27 T. Cosby, U. Kapoor, J. K. Shah and J. Sangoro, Mesoscale Organization and Dynamics in Binary Ionic Liquid Mixtures, J. Phys. Chem. Lett., 2019, 10, 6274-6280.

28 A. P. Abbott, G. Capper, D. L. Davies, R. K. Rasheed and V. Tambyrajah, Novel solvent properties of choline chloride/urea mixtures, Chem. Commun., 2003, 70-71. 
29 F. S. Mjalli and O. U. Ahmed, Physical properties and intermolecular interaction of eutectic solvents binary mixtures: reline and ethaline, AsiaPacific J. Chem. Eng., 2016, 11, 549-557.

30 S. B. Phadtare and G. S. Shankarling, Halogenation reactions in biodegradable solvent: Efficient bromination of substituted 1-aminoanthra9,10-quinone in deep eutectic solvent (choline chloride: Urea), Green Chem., 2010, 12, 458-46.

31 D. Lindberg, M. de la Fuente Revenga and M. Widersten, Deep eutectic solvents (DESs) are viable cosolvents for enzyme-catalyzed epoxide hydrolysis, J. Biotechnol., 2010, 147, 169-171.

32 X. Li, M. Hou, B. Han, X. Wang and L. Zou, Solubility of CO2 in a choline chloride + urea eutectic mixture, J. Chem. Eng. Data, 2008, 53, 548-550.

33 W. C. Su, D. S. H. Wong and M. H. Li, Effect of water on solubility of carbon dioxide in (aminomethanamide + 2-hydroxy-N,N,Ntrimethylethanaminium chloride), J. Chem. Eng. Data, 2009, 54, 1951-1955.

34 M. Hayyan, F. S. Mjalli, M. A. Hashim and I. M. AlNashef, A novel technique for separating glycerine from palm oil-based biodiesel using ionic liquids, Fuel Process. Technol., 2010, 91, 116-120.

35 Q. Zhang, K. De Oliveira Vigier, S. Royer and F. Jérôme, Deep eutectic solvents: Syntheses, properties and applications, Chem. Soc. Rev., 2012, 41, 7108-7146.

36 Y. S. Liao, P. Y. Chen and I. W. Sun, Electrochemical study and recovery of $\mathrm{Pb}$ using 1:2 choline chloride/urea deep eutectic solvent: A variety of $\mathrm{Pb}$ species $\mathrm{PbSO} 4, \mathrm{PbO} 2$, and $\mathrm{PbO}$ exhibits the analogous thermodynamic behavior, Electrochim. Acta, 2016, 214, 265-275.

37 M. Bučko, D. Culliton, A. J. Betts and J. B. Bajat, The electrochemical deposition of $\mathrm{Zn}-\mathrm{Mn}$ coating from choline chloride-urea deep eutectic solvent, Trans. IMF, 2017, 95, 60-64.

38 H. G. Morrison, C. C. Sun and S. Neervannan, Characterization of thermal behavior of deep eutectic solvents and their potential as drug solubilization vehicles, Int. J. Pharm., 2009, 378, 136-139.

39 H. Monhemi, M. R. Housaindokht, A. A. Moosavi-Movahedi and M. R. Bozorgmehr, in Phys. Chem. Chem. Phys., 2014, vol. 16, pp. 14882-14893. 
40 A. Maity, S. Sarkar, L. Theeyancheri and R. Chakrabarti, Choline Chloride as a Nano-Crowder Protects HP-36 from Urea-Induced Denaturation: Insights from Solvent Dynamics and Protein-Solvent Interactions, ChemPhysChem, 2020, 21, 552-567.

41 S. Sarkar, S. Ghosh and R. Chakrabarti, Ammonium based stabilizers effectively counteract urea-induced denaturation in a small protein: Insights from molecular dynamics simulations, RSC Adv., 2017, 7, 52888-52906.

42 S. L. Perkins, P. Painter and C. M. Colina, Molecular dynamic simulations and vibrational analysis of an ionic liquid analogue, J. Phys. Chem. B, 2013, 117, 10250-10260.

43 M. Campetella, E. Bodo, R. Caminiti, A. Martino, F. D'apuzzo, S. Lupi and L. Gontrani, Interaction and dynamics of ionic liquids based on choline and amino acid anions, J. Chem. Phys, 2015, 142, 234502.

44 X.-D. Hou, Q.-P. Liu, T. J. Smith, N. Li and M.-H. Zong, Evaluation of Toxicity and Biodegradability of Cholinium Amino Acids Ionic Liquids, PLoS One, 2013, 8, e59145.

45 D. Kumar Sahoo, S. Jena, K. Devi Tulsiyan, J. Dutta, S. Chakrabarty and H. S. Biswal, Amino-Acid-Based Ionic Liquids for the Improvement in Stability and Activity of Cytochrome c: A Combined Experimental and Molecular Dynamics Study, 2019, 123, 10100-10109.

46 S. Pal and S. Paul, Understanding the Role of Reline, a Natural DES, on Temperature-Induced Conformational Changes of C-Kit G-Quadruplex DNA: A Molecular Dynamics Study, J. Phys. Chem. B, 2020, 124, $3123-$ 3136.

47 S. Kaur, S. Sharma and H. K. Kashyap, Bulk and interfacial structures of reline deep eutectic solvent: A molecular dynamics study, J. Chem. Phys., 2017, 147, 194507.

48 H. Sun, Y. Li, X. Wu and G. Li, Theoretical study on the structures and properties of mixtures of urea and choline chloride, J. Mol. Model., 2013, 19, 2433-2441.

49 E. Posada, N. López-Salas, R. J. Jiménez Riobóo, M. L. Ferrer, M. C. Gutiérrez and F. Del Monte, Reline aqueous solutions behaving as liquid mixtures of H-bonded co-solvents: Microphase segregation and formation of co-continuous structures as indicated by Brillouin and $1 \mathrm{H} \mathrm{NMR}$ 
spectroscopies, Phys. Chem. Chem. Phys., 2017, 19, 17103-17110.

50 D. Shah and F. S. Mjalli, Effect of water on the thermo-physical properties of Reline: An experimental and molecular simulation based approach, Phys. Chem. Chem. Phys., 2014, 16, 23900-23907.

51 C. D’Agostino, L. F. Gladden, M. D. Mantle, A. P. Abbott, E. I. Ahmed, A. Y. M. Al-Murshedi and R. C. Harris, Molecular and ionic diffusion in aqueous-deep eutectic solvent mixtures: Probing inter-molecular interactions using PFG NMR, Phys. Chem. Chem. Phys., 2015, 17, 15297-15304.

52 O. S. Hammond, D. T. Bowron and K. J. Edler, The Effect of Water upon Deep Eutectic Solvent Nanostructure: An Unusual Transition from Ionic Mixture to Aqueous Solution, Angew. Chemie - Int. Ed., 2017, 56, 97829785.

53 E. O. Fetisov, D. B. Harwood, I. F. W. Kuo, S. E. E. Warrag, M. C. Kroon, C. J. Peters and J. I. Siepmann, First-Principles Molecular Dynamics Study of a Deep Eutectic Solvent: Choline Chloride/Urea and Its Mixture with Water, J. Phys. Chem. B, 2018, 122, 1245-1254.

54 P. Kumari, Shobhna, S. Kaur and H. K. Kashyap, Influence of Hydration on the Structure of Reline Deep Eutectic Solvent: A Molecular Dynamics Study, ACS Omega, 2018, 3, 15246-15255.

55 S. Kaur, A. Malik and H. K. Kashyap, Anatomy of Microscopic Structure of Ethaline Deep Eutectic Solvent Decoded through Molecular Dynamics Simulations, J. Phys. Chem. B, 2019, 123, 8291-8299.

56 D. Bandyopadhyay, S. Mohan, S. K. Ghosh and N. Choudhury, Correlation of structural order, anomalous density, and hydrogen bonding network of liquid water, J. Phys. Chem. B, 2013, 117, 8831-8843.

57 E. A. Oprzeska-Zingrebe and J. Smiatek, Aqueous Mixtures of Urea and Trimethylamine-N-oxide: Evidence for Kosmotropic or Chaotropic Behavior?, J. Phys. Chem. B, 2019, 123, 4415-4424.

58 C. J. Sahle, M. A. Schroer, I. Juurinen and J. Niskanen, Influence of TMAO and urea on the structure of water studied by inelastic X-ray scattering, Phys. Chem. Chem. Phys., 2016, 18, 16518-16526.

59 Y. L. A. Rezus and H. J. Bakker, Observation of immobilized water molecules around hydrophobic groups, Phys. Rev. Lett., 2007, 99, 148301. 
60 A. Panuszko, P. Bruździak, J. Zielkiewicz, D. Wyrzykowski and J. Stangret, Effects of urea and trimethylamine-N-oxide on the properties of water and the secondary structure of hen egg white lysozyme, J. Phys. Chem. B, 2009, 113, 14797-14809.

61 J. Hunger, K. J. Tielrooij, R. Buchner, M. Bonn and H. J. Bakker, Complex formation in aqueous trimethylamine-N-oxide (TMAO) solutions, J. Phys. Chem. B, 2012, 116, 4783-4795.

62 L. Larini and J. E. Shea, Double resolution model for studying TMAO/water effective interactions, J. Phys. Chem. B, 2013, 117, 13268-13277.

63 L. Knake, G. Schwaab, K. Kartaschew and M. Havenith, Solvation Dynamics of Trimethylamine N-Oxide in Aqueous Solution Probed by Terahertz Spectroscopy, J. Phys. Chem. B, 2015, 119, 13842-13851.

64 S. Indra and R. Biswas, Is dynamic heterogeneity of water in presence of a protein denaturing agent different from that in presence of a protein stabilizer? A molecular dynamics simulation study, J. Chem. Sci., 2016, 128, 1943-1954.

65 S. Paul and G. N. Patey, Structure and interaction in aqueous urea Trimethylamine-N-oxide solutions, J. Am. Chem. Soc., 2007, 129, 44764482.

66 J. Smiatek, Osmolyte effects: Impact on the aqueous solution around charged and neutral spheres, J. Phys. Chem. B, 2014, 118, 771-782.

67 Y. L. A. Rezus and H. J. Bakker, Effect of urea on the structural dynamics of water, Proc. Natl. Acad. Sci. U. S. A., 2006, 103, 18417-18420.

68 D. Bandyopadhyay, S. Mohan, S. K. Ghosh and N. Choudhury, Molecular Dynamics Simulation of Aqueous Urea Solution: Is Urea a Structure Breaker?, 2014, 118, 11757-11768.

69 D. Ojha and A. Chandra, Urea in Water: Structure, Dynamics, and Vibrational Echo Spectroscopy from First-Principles Simulations, J. Phys. Chem. B, 2019, 123, 3325-3336.

70 G. E. Walrafen, Raman Spectral Studies of the Effects of Urea and Sucrose on Water Structure, J. Chem. Phys., 1966, 44, 3726-3727.

71 B. Jana, S. Pal and B. Bagchi, Enhanced tetrahedral ordering of water molecules in minor grooves of DNA: Relative role of DNA rigidity, 
nanoconfinement, and surface specific interactions, J. Phys. Chem. B, 2010, 114, 3633-3638.

72 S. Daschakraborty, How do glycerol and dimethyl sulphoxide affect local tetrahedral structure of water around a nonpolar solute at low temperature? Importance of preferential interaction, J. Chem. Phys., 2018, 148, 134501.

73 D. Nayar, M. Agarwal and C. Chakravarty, Comparison of tetrahedral order, liquid state anomalies, and hydration behavior of mTIP3P and TIP4P water models, J. Chem. Theory Comput., 2011, 7, 3354-3367.

74 V. Dubey, S. Erimban, S. Indra and S. Daschakraborty, Understanding the Origin of the Breakdown of the Stokes-Einstein Relation in Supercooled Water at Different Temperature-Pressure Conditions, 2019, 123, 1008910099.

75 B. Hess, C. Kutzner, D. Van Der Spoel and E. Lindahl, GROMACS 4: Algorithms for highly efficient, load-balanced, and scalable molecular simulation, J. Chem. Theory Comput., 2008, 4, 435-447.

76 W. L. Jorgensen and J. Tirado-Rives, The OPLS Potential Functions for Proteins. Energy Minimizations for Crystals of Cyclic Peptides and Crambin, J. Am. Chem. Soc., 1988, 110, 1657-1666.

77 S. Ghosh, S. Dey, M. Patel and R. Chakrabarti, Can an ammonium-based room temperature ionic liquid counteract the urea-induced denaturation of a small peptide?, Phys. Chem. Chem. Phys., 2017, 19, 7772-7787.

78 B. Doherty and O. Acevedo, OPLS Force Field for Choline Chloride-Based Deep Eutectic Solvents, J. Phys. Chem. B, 2018, 122, 9982-9993.

79 S. Zhu, Validation of the Generalized Force Fields GAFF, CGenFF, OPLSAA, and PRODRGFF by Testing against Experimental Osmotic Coefficient Data for Small Drug-Like Molecules, J. Chem. Inf. Model., 2019, 59, 42394247.

80 A. Yadav and S. Pandey, Densities and viscosities of (choline chloride + urea) deep eutectic solvent and its aqueous mixtures in the temperature range 293.15 K to 363.15 K, J. Chem. Eng. Data, 2014, 59, 2221-2229.

81 P. Mark and L. Nilsson, Structure and dynamics of the TIP3P, SPC, and SPC/E water models at $298 \mathrm{~K}$, J. Phys. Chem. A, 2001, 105, 9954-9960.

82 S. S. Petrova and A. D. Solov'Ev, The Origin of the Method of Steepest 
Descent, Hist. Math., 1997, 24, 361-375.

83 G. Bussi, D. Donadio and M. Parrinello, Canonical sampling through velocity rescaling, J. Chem. Phys., 2007, 126, 014101.

84 M. Parrinello and A. Rahman, Polymorphic transitions in single crystals: A new molecular dynamics method, J. Appl. Phys., 1981, 52, 7182-7190.

85 M.P. Allen and D. J. Tildesley, Computer Simulation of Liquids: Second Edition, Oxford University Press, 2017.

86 H. C. Andersen, Rattle: A 'velocity' version of the shake algorithm for molecular dynamics calculations, J. Comput. Phys., 1983, 52, 24-34.

87 T. Darden, D. York and L. Pedersen, Particle mesh Ewald: An N $\log (\mathrm{N})$ method for Ewald sums in large systems, J. Chem. Phys., 1993, 98, 1008910092.

88 W. Humphrey, A. Dalke and K. Schulten, VMD: Visual molecular dynamics, J. Mol. Graph., 1996, 14, 33-38.

89 G. N. I. Clark, C. D. Cappa, J. D. Smith, R. J. Saykally and T. Head-Gordon, The structure of ambient water, Mol. Phys., 2010, 108, 1415-1433.

90 L. Hernández De La Peña and P. G. Kusalik, Quantum effects in liquid water and ice: Model dependence, J. Chem. Phys., 2006, 125, 054512.

91 P. Banerjee, S. Yashonath and B. Bagchi, Coupled jump rotational dynamics in aqueous nitrate solutions, J. Chem. Phys., 2016, 145, 234502.

92 N. Galamba, Water's structure around hydrophobic solutes and the iceberg model, J. Phys. Chem. B, 2013, 117, 2153-2159.

93 N. Giovambattista, P. G. Debenedetti, F. Sciortino and H. E. Stanley, Structural order in glassy water, Phys. Rev. E - Stat. Nonlinear, Soft Matter Phys., 2005, 71, 061505.

94 S. Chatterjee, P. G. Debenedetti, F. H. Stillinger and R. M. Lynden-Bell, A computational investigation of thermodynamics, structure, dynamics and solvation behavior in modified water models, J. Chem. Phys., 2008, 128, 124511.

95 P. Chau and A. J. Hardwick, A new order parameter for tetrahedral configurations, Mol. Phys., 1998, 93, 511-518. 
96 J. R. Errington and P. G. Debenedetti, Relationship between structural order and the anomalies of liquid water, Nature, 2001, 409, 318-321.

97 L. Aggarwal and P. Biswas, Hydration Water Distribution around Intrinsically Disordered Proteins, J. Phys. Chem. B, 2018, 122, 4206-4218.

98 M. D. Elola and B. M. Ladanyi, Computational study of structural and dynamical properties of formamide-water mixtures, J. Chem. Phys., 2006, 125, 184506.

99 M. C. Stumpe and H. Grubmüller, Aqueous urea solutions: Structure, energetics, and urea aggregation, J. Phys. Chem. B, 2007, 111, 6220-6228.

100 A. Luzar and D. Chandler, Hydrogen-bond kinetics in liquid water, Nature, 1996, 379, 55-57.

101 Z. Lu, M. Lanagan, E. Manias and D. D. MacDonald, Two-port transmission line technique for dielectric property characterization of polymer electrolyte membranes, J. Phys. Chem. B, 2009, 113, 13551-13559.

102 S. Shaukat, M. V. Fedotova, S. E. Kruchinin, M. Bešter-Rogač, Č. Podlipnik and R. Buchner, Hydration and ion association of aqueous choline chloride and chlorocholine chloride, Phys. Chem. Chem. Phys., 2019, 21, 1097010980 .

103 K. R. Wilson, B. S. Rude, T. Catalane, R. D. Schaller, J. G. Tobin, D. T. Co and R. J. Saykally, X-ray spectroscopy of liquid water microjets, J. Phys. Chem. B, 2001, 105, 3346-3349.

104 M. Aryafard, A. Karimi, A. R. Harifi-Mood and B. Minofar, Molecular Dynamics Simulations, Solvatochromic Parameters, and Preferential Solvation in Aqueous Solutions of Ethaline, Ethylene Glycol, and Choline Chloride, J. Chem. Eng. Data, 2020, 65, 4556-4566.

105 M. Aryafard, M. Abbasi, D. Řeha, A. R. Harifi-Mood and B. Minofar, Experimental and theoretical investigation of solvatochromic properties and ion solvation structure in DESs of reline, glyceline, ethaline and their mixtures with PEG 400, J. Mol. Liq., 2019, 284, 59-67.

106 A. R. Harifi-Mood, R. Ghobadi, S. Matić, B. Minofar and D. Řeha, Solvation analysis of some Solvatochromic probes in binary mixtures of reline, ethaline, and glyceline with DMSO, J. Mol. Liq., 2016, 222, 845-853.

107 S. Pal and S. Paul, Effect of Hydrated and Nonhydrated Choline Chloride- 
Urea Deep Eutectic Solvent (Reline) on Thrombin-Binding G-quadruplex Aptamer (TBA): A Classical Molecular Dynamics Simulation Study, J. Phys. Chem. C, 2019, 123, 11686-11698. 